\title{
Terrestrial monitoring of a radio telescope reference point using comprehensive uncertainty budgeting
}

\author{
Investigations during CONT14 at the Onsala Space Observatory
}

\author{
Michael Lösler $^{1}$ • Rüdiger Haas ${ }^{2}$ • Cornelia Eschelbach ${ }^{1}$
}

Received: 23 June 2015 / Accepted: 14 January 2016 / Published online: 12 February 2016

(C) The Author(s) 2016. This article is published with open access at Springerlink.com

\begin{abstract}
During the 15-day-long global very long baseline interferometry campaign CONT14, a terrestrial monitoring campaign was carried out at the Onsala Space Observatory. The goal of these efforts was to monitor the reference point of the Onsala $20 \mathrm{~m}$ radio telescope during normal telescope operations. Parts of the local site network as well as a number of reflectors that were mounted on the $20 \mathrm{~m}$ radio telescope were observed in an automated and continual way using the in-house-developed software package HEIMDALL. The analysis of the observed data was performed using a new concept for a coordinate-based network adjustment to allow the full adjustment process in a true Cartesian global reference frame. The Akaike Information Criterion was used to select the preferable functional model for the network adjustment. The comprehensive stochastic model of this network adjustment process considers over 25 parameters, and, to describe the persistence of the observations performed during the monitoring with a very high measurement frequency, includes also time-dependent covariances. In total 15 individual solutions for the radio telescope reference point were derived, based on monitoring observations during the normal
\end{abstract}

Michael Lösler

michael.loesler@fb1.fra-uas.de

Rüdiger Haas

rudiger.haas@chalmers.se

Cornelia Eschelbach

cornelia.eschelbach@fb1.fra-uas.de

1 Faculty 1: Architecture, Civil Engineering, Geomatic, Laboratory for Industrial Metrology, Frankfurt University of Applied Sciences, Nibelungenplatz 1, 60318 Frankfurt am Main, Germany

2 Department of Earth and Space Sciences, Chalmers University of Technology, Onsala Space Observatory, 43992 Onsala, Sweden operation of the radio telescope. Since the radio telescope was moving continually, the influence of timing errors was studied and considered in the adjustment process. Finally, recursive filter techniques were introduced to combine the 15 individual solutions. Accuracies at the sub-millimeter level could be achieved for the radio telescope reference point. Thus, the presented monitoring concept fulfills the requirement proposed by the global geodetic observing system.

Keywords Radio telescope - VLBI · Reference point determination · Monitoring - Uncertainty · Bundle adjustment . Least-squares analysis · Akaike Information Criterion

\section{Introduction}

The increasingly complex and environmentally stressed world requires accurate global Earth observations to support decision making for a sustainable development and the benefit and survival of human society. The prerequisite for such global Earth observations is an accurate and reliable global terrestrial reference frame. Recently, the United Nations (UN) highlighted the importance of a global terrestrial reference frame and adopted a corresponding UN resolution on a global geodetic reference frame for sustainable development (United Nations 2015).

A global geodetic reference frame is realized by combining results from a variety of space geodetic techniques. It is published by the International Earth Rotation and Reference Systems Service (IERS) as the International Terrestrial Reference Frame (ITRF), see e.g. Altamimi et al. (2011). To combine the measurements performed by the different space geodetic techniques, so-called co-location stations that host equipment for more than one technique are crucial. The local-tie vectors between the reference points of the space 

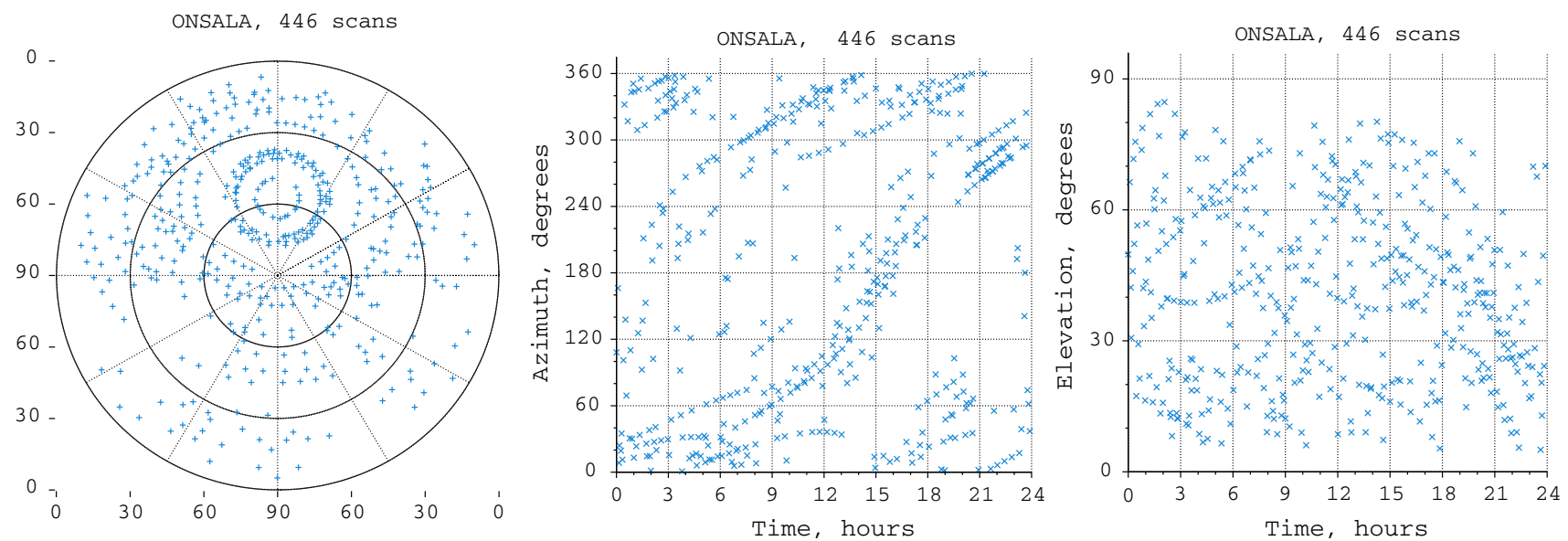

Fig. 1 Left: Local sky coverage at Onsala during one of the $24 \mathrm{~h}$ long CONT14 sessions. Middle: Azimuth as a function of time during this session. Right: Elevation as a function of time during this session

geodetic equipment need to be known with high accuracy. Rothacher et al. (2009) advise that measurements of localtie vectors should be performed with $1 \mathrm{~mm}$ accuracy in a fully automated way and on an almost continuous basis, since local ties may change over time. The determination of localtie vectors involves determining the reference points of the space geodetic equipment as, e.g. radio telescopes used for VLBI observations. In general, the determination of the reference point of a radio telescope is a demanding engineering task. However, it is a particular challenge for the upcoming VGOS (VLBI Global Observing System) (Petrachenko et al. 2009) that prepares for so-called $24 / 7$ operations, i.e. $24 \mathrm{~h}$ a day, 7 days a week. The VGOS radio telescopes will be in operation continually and there will hardly be time available for dedicated survey campaigns to determine radio telescope reference points and local-tie vectors.

During a geodetic VLBI session, a network of several radio telescopes observes a number of extragalactic radio sources to achieve interferometric measurements [e.g. Sovers et al. (1998)]. The radio telescopes are located far away from each other, often on different tectonic plates, and thus form very long baselines. The telescopes simultaneously observe identical radio sources, one at the time. During the observations, the radio telescopes need to compensate for the rotation of the Earth to keep tracking the radio sources. The radio telescopes have, therefore, to continually change their local azimuth and elevation orientation slightly. The Earth rotates by roughly 15 arcsec per second, and this rotational velocity maps into the azimuth and elevation speed of the radio telescopes. The exact tracking speed in azimuth and elevation that is necessary to follow a radio source depends on the location of the particular radio telescope and the local azimuth and elevation direction of the radio source. For example, for a radio telescope on the equator and a radio source in the equatorial plane the elevation speed would be 15 arcsec per second while there would not be any movement in azimuth. The observation of one particular radio source usually takes between about 15 seconds and 5 minutes. The exact observation time is calculated in advance during the preparation of the observing plan, the so-called schedule, and is a function of the known signal strength of the particular radio source to be observed and the sensitivity of the involved radio telescopes. Once a radio source has been observed, the radio telescopes point at the next radio source in the schedule, thus they change from one tracking direction to another. The movement between two tracking directions occurs at relatively high speed, for example, at the Onsala $20 \mathrm{~m}$ telescope with $3^{\circ} / \mathrm{s}$ in azimuth and $1 \%$ in elevation. The arc length between different observing directions usually is rather large to achieve beneficial geometrical conditions for the analysis of the VLBI data. During a 24-hlong observing session, in total on the order of 400-500 radio source observations in different azimuth/elevation directions are performed at a single radio telescope. Thus, the local sky at a radio telescope is covered rather homogeneously with observations in many different azimuth/elevation directions, which is a benefit for the analysis of the VLBI data. In general, each azimuth/elevation direction during a 24-h-long observing session is unique and not covered twice. The involved radio telescopes are continually changing their azimuth and elevation orientation, either while observing (tracking) a particular radio source and compensating for Earth rotation, or switching (slewing) between different radio sources.

The left graph in Fig. 1 depicts as an example the local sky coverage, i.e. the directions that the Onsala radio telescope was pointing at, during one of the 15 sessions of $24 \mathrm{~h}$ observations length that were observed during CONT14. ${ }^{1}$

\footnotetext{
${ }^{1}$ Continuous VLBI Campaign 2014 http://ivscc.gsfc.nasa.gov/ program/cont14/.
} 
The corresponding azimuth and elevation of the radio source observations as a function of time are presented in middle and right graph of Fig. 1, respectively. In total, the Onsala radio telescope observed radio sources in 446 different azimuth/elevation directions during these $24 \mathrm{~h}$. More than $57,16,10$ and $5 \%$ of the radio source observations had an observation length of $15,30,45$ and $60 \mathrm{~s}$, respectively. The remaining $12 \%$ of the observations were longer than $1 \mathrm{~min}$, where one single observation was 3 minutes long. The minimum and maximum arc lengths between two observing directions were $10^{\circ}$ and $180^{\circ}$, and about $13 \%$ of the arc lengths were $60^{\circ}$.

Methods and routines have been developed to allow the determination and monitoring of the radio telescope reference point during ongoing operations (Lösler et al. 2013). The current work extends Lösler et al. (2013) considerably by implementing a comprehensive stochastic model that takes more than 25 uncertainty parameters into account, by applying a coordinate-based bundle adjustment in combination with a recursive filter, and by providing the results for the radio telescope reference point directly in a Cartesian global reference frame. The uncertainty budgeting is derived according to the DIN 1319 (1995) taken into account uncertainties that are derived by statistical analysis as well as uncertainties that are derived by non-statistical approaches. This innovative and advanced monitoring approach was applied for the first time during the 15-day-long continuous VLBI campaign in 2014, CONT14. Figure 2 depicts a simplified flowchart of the monitoring and analysis process of the in-house developed monitoring software HEIMDALL ${ }^{2}$ to have an impression of the complexity of the automated IVS reference point determination. The work presented in this paper focuses on the components that are marked by black solid boxes. To learn more about the monitoring components of the HEIMDALL system the interested reader is invited to read the prior work of Lösler et al. (2013).

Section 2 describes the general model used to determine the reference point of a radio telescope. The analysis strategy using the Gauß-Helmert model is explained briefly in Sect. 3, followed by a description of the coordinatebased bundle adjustment in Sect. 4. To evaluate a preferable functional model for the bundle adjustment, Sect. 4.2 introduces the information criteria technique to avoid an overparametrization. The ingredients of the complete stochastic model are explained in detail in Sects. 4-7. Section 8 explains the recursive parameter estimation, followed by a description of the actual monitoring performed during CONT14 in Sect. 9. The data analysis is explained in Sect. 10, followed by the presentation and discussion of the results in Sect. 11. Section 12 finally concludes the paper. The Appendix explains

\footnotetext{
$\overline{2}$ High-end interface for monitoring and spatial data analysis using L2Norm.
}

the derivation of a compensation model for polar measurement instruments.

\section{Radio telescope reference point determination}

The International VLBI Service for Geodesy and Astrometry (IVS) defines the geometrical reference point of an azimuthelevation type radio telescope as the intersection point of the azimuth and elevation axes (Nothnagel 2009). Depending on the design and/or actual construction of the radio telescope, an axis-offset may exist. In this case, the reference point is defined by the orthogonal projection of the elevation axis onto the azimuth axis. Usually, the reference point cannot be materialized for direct measurements, and therefore indirect survey methods need to be applied [e.g. Eschelbach and Haas (2003), Sarti et al. (2004), Dawson et al. (2007), Leinen et al. (2007), Lanotte et al. (2008), Lösler (2008), Kallio and Poutanen (2012), Li et al. (2014), Ning et al. (2015)].

To determine the reference point during normal telescope operations, a general mathematical model has been proposed by Lösler (2009). This approach includes the radio telescope azimuth and elevation angles as additional observations. This model can be interpreted as a transformation between the ground-fixed reference system and the radio telescope system

$\mathbf{P}_{\mathrm{Obs}}=\mathbf{P}_{\mathrm{RP}}+\mathbf{R}_{\theta}^{x} \mathbf{R}_{\phi}^{y} \mathbf{R}_{\alpha-\mathrm{O}_{\alpha}}^{z} \mathbf{R}_{\psi}^{y}\left(\mathbf{E}_{\alpha, \epsilon}+\mathbf{R}_{\epsilon}^{x} \mathbf{P}_{\mathrm{Tel}}\right)$.

In the above equation $\alpha$ and $\epsilon$ denote the azimuth and elevation angles of the radio telescope, respectively. The vector $\mathbf{P}_{\text {Obs }}$ represents the observed position in the ground-fixed system and $\mathbf{P}_{\mathrm{Tel}}$ denotes the corresponding position in the radio telescope system. Depending on the definition of the site network, $\mathbf{P}_{\mathrm{Obs}}$ can be a local topocentric position or even a global geocentric one. The position $\mathbf{P}_{\mathrm{Tel}}$ is defined by the radial distances to the elevation-axis $a_{\epsilon}$, the radial distances to the azimuth-axis $b_{\alpha}$ and an elevation orientation angle $\mathrm{O}_{\epsilon}$

$\mathbf{P}_{\mathrm{Tel}}=\mathbf{R}_{-\mathrm{O}_{\epsilon}}^{x}\left(\begin{array}{c}b_{\alpha} \\ a_{\epsilon} \\ 0\end{array}\right)$.

The non-orthogonality between the azimuth and elevation axes is parametrized by the angle $\psi$, and the axis-offset $e_{\alpha, \epsilon}$ is given by $\mathbf{E}_{\alpha, \epsilon}=\left(0 e_{\alpha, \epsilon} 0\right)^{\mathrm{T}}$. Both coordinate systems become congruent by the rotation sequence $\mathbf{R}_{\theta}^{x} \mathbf{R}_{\phi}^{y} \mathbf{R}_{-\mathrm{O}_{\alpha}}^{z}$ with the angles $\theta, \phi$ and $\mathrm{O}_{\alpha}$, respectively, and a final translation with vector $\mathbf{P}_{\mathrm{RP}}$ that represents the reference point which is defined by the IVS w.r.t. the ground-fixed site network. Since the observations and the unknown parameters in Eq. 1 cannot be separated from each other, it is necessary to apply a general least-squares adjustment, also known as Gauß-Helmert model (see Sect. 3), to solve Eq. 1. To study the detailed 
Fig. 2 Simplified flowchart of the monitoring and analysis process of the HEIMDALL system. The grey-dashed boxes represent components that are out of scope of the current work, and most of these are discussed in detail by Lösler et al. (2013). The black solid boxes represent components that are discussed in the current work

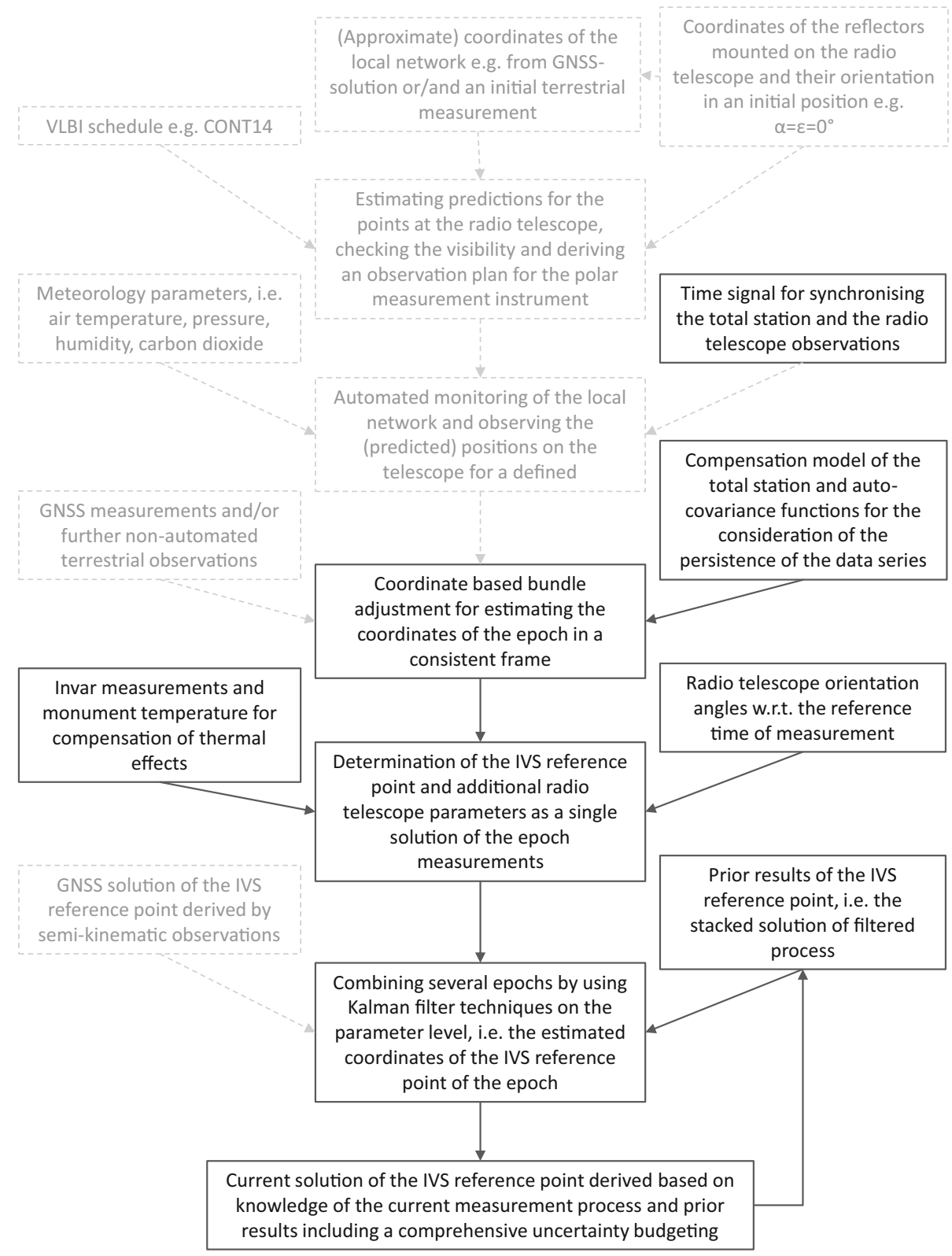

derivation and additional graphical material of the reference point determination model, the interested reader is referred to Lösler (2008) and Lösler (2009).

Temperature variations affect the telescope monument and cause a height variation $\Delta h_{\mathrm{RP}}$ of the radio telescope [e.g. Haas et al. (1999), Wresnik et al. (2007)]. In accordance with the IVS convention by Nothnagel (2009), the height variation can be expressed as

$$
\Delta h_{\mathrm{RP}}\left(\Delta T_{i}\right)=\left(\gamma_{S} h_{S}+\gamma_{F} h_{F}\right) \Delta T_{i}
$$

Here $h_{S}$ is the height of the reference point with respect to the foundation of the telescope, $h_{F}$ is the height of the foundation, and $\gamma_{S}$ and $\gamma_{F}$ are the thermal expansion coefficients for the material of the telescope and the foundation, respectively. The difference between the temperature of the radio telescope structure $T_{i}$ and a reference temperature $T_{0}$ is denoted by $\Delta T_{i}=T_{i}-T_{0}$. Similar to the height component, the parameters $a_{\epsilon}$ and $b_{\alpha}$ (see Eq. 2) are also affected and should be corrected for thermal effects [cf. Lösler et al. (2013)]

$$
\begin{aligned}
\Delta a_{\epsilon}\left(\Delta T_{i}\right) & =\gamma_{S} a_{\epsilon} \Delta T_{i}, \\
\Delta b_{\alpha}\left(\Delta T_{i}\right) & =\gamma_{S} b_{\alpha} \Delta T_{i} .
\end{aligned}
$$

Depending on the geodetic datum, the local (vertical) height component might be aligned to the $z_{\mathrm{RP}}$-coordinate of the reference point. In this case, the correction of the thermal expansion can directly be applied to the $z \mathrm{RP}$-component 
of the reference point. However, if a geodetic datum is chosen where the $z_{\mathrm{RP}}$-coordinate is not aligned with the local (vertical) height component, e.g. using a global geocentric reference frame, the rotation between the telescope system and the local ground-fixed system has to be taken into account. Then the height variation has to be split up into the coordinate components of $\mathbf{P}_{\mathrm{RP}}$ by

$$
\left(\begin{array}{c}
\Delta x_{\mathrm{RP}} \\
\Delta y_{\mathrm{RP}} \\
\Delta z_{\mathrm{RP}}
\end{array}\right)_{\text {Global }}=\mathbf{R}_{\theta}^{x} \mathbf{R}_{\phi}^{y}\left(\begin{array}{c}
0 \\
0 \\
\Delta h_{\mathrm{RP}}
\end{array}\right)_{\text {Local }} .
$$

\section{Brief description of the Gauß-Helmert model}

The Gauß-Helmert model (GHM) is known as the general least-squares method, which solves adjustment problems with condition equations. The functional model of the GHM connects the unknown parameters $\mathbf{x}$, the observations $\mathbf{I}$ and their random errors $\mathbf{v}$ by $n$ differentiable condition equations $\Psi(\mathbf{x}, \mathbf{l}+\mathbf{v})=0$ [cf. Neitzel (2010), Koch (2014)]. By providing appropriate a priori values $\mathbf{x}_{\mathbf{0}}$ and $\mathbf{v}_{\mathbf{0}}$, the normal equation system can be written as

$$
\left[\begin{array}{c}
\mathbf{k} \\
\mathbf{d x}
\end{array}\right]=-\left[\begin{array}{cc}
\mathbf{B} \mathbf{Q}_{\boldsymbol{I I}} \mathbf{B}^{\mathrm{T}} & \mathbf{A} \\
\mathbf{A}^{\mathrm{T}} & \mathbf{0}
\end{array}\right]^{-1}\left[\begin{array}{c}
-\mathbf{B} \mathbf{v}_{\mathbf{0}}+\Psi\left(\mathbf{x}_{\mathbf{0}}, \mathbf{v}_{\mathbf{0}}\right) \\
\mathbf{0}
\end{array}\right]
$$

Here, the design matrices $\mathbf{A}=\left.\frac{\partial \Psi}{\partial \mathbf{x}}\right|_{\mathbf{x}_{\mathbf{0}}, \mathbf{l}_{\mathbf{0}}}$ and $\mathbf{B}=\left.\frac{\partial \Psi}{\partial \mathbf{l}}\right|_{\mathbf{x}_{\mathbf{0}}, \mathbf{l}_{\mathbf{0}}}$ consist of the partial derivatives of the linearized functional model with respect to the unknown parameters $\mathbf{x}$ and the observations I, respectively. The vector of Lagrange multipliers is denoted by $\mathbf{k}$, and the vector of misclosures is defined by $\mathbf{w}=-\mathbf{B} \mathbf{v}_{\mathbf{0}}+\Psi\left(\mathbf{x}_{\mathbf{0}}, \mathbf{l}_{\mathbf{0}}\right)$. The corrections $\mathbf{d x}$ to the a priori values $\mathbf{x}_{\mathbf{0}}$ are determined by solving the normal equation system and the estimated solutions of $\mathbf{x}=\mathbf{x}_{\mathbf{0}}+\mathbf{d x}$ and $\mathbf{l}=\mathbf{l}_{\mathbf{0}}+\mathbf{v}=\mathbf{l}_{\mathbf{0}}+\mathbf{Q}_{\mathbf{I I}} \mathbf{B}^{\mathrm{T}} \mathbf{k}$ are introduced as new approximations to the next iteration step. This procedure is repeated until the system of equations converges. The variance-covariance matrix $\mathbf{Q}_{\mathrm{II}}$ in Eq. 7 represents the stochastic model of the observations.

Whereas the vector I contains the observed positions $\mathbf{P}_{\mathrm{Obs}, i}$ as well as the related azimuth angles $\alpha_{i}$ and elevation angles $\epsilon_{i}$, the vector of unknown parameters $\mathbf{x}$ includes the reference point $\mathbf{P}_{\mathrm{RP}}$, the axis-offset $e_{\alpha, \epsilon}$, the correction angles that compensate for misalignment of the axes $(\psi, \theta$ and $\phi$ ), an azimuth zero-point correction $\mathrm{O}_{\alpha}$ as well as the additional point depending parameters $a_{\epsilon}, b_{\alpha}$ and $\mathrm{O}_{\epsilon}$ of $\mathbf{P}_{\mathrm{Tel}}$. A detailed description of the Gauß-Helmert model in context of the derived reference point determination model (see Eq. 1) is given by Lösler (2009).

Applying the law of uncertainty propagation, the stochastic dependencies between the estimated parameters $\mathbf{x}$ and the observations I can be derived by
$\mathbf{Q}_{\mathbf{l x}}=-\mathbf{Q}_{\mathrm{ll}} \mathbf{B}^{\mathrm{T}} \mathbf{Q}_{\mathbf{w w}}^{-1} \mathbf{A} \mathbf{Q}_{\mathbf{x x}}$

Here $\mathbf{Q}_{\mathbf{x x}}=\left(\mathbf{A}^{\mathrm{T}} \mathbf{Q}_{\mathbf{w w}}^{-\mathbf{1}} \mathbf{A}\right)^{-\mathbf{1}}$ denotes the estimated variancecovariance matrix of the unknown parameters $\mathbf{x}$ and $\mathbf{Q}_{\mathbf{w w}}=\mathbf{B} \mathbf{Q}_{\boldsymbol{l l}} \mathbf{B}^{\mathrm{T}}$ is the variance-covariance matrix of the misclosures $\mathbf{w}$ [e.g. Höpcke (1980, p. 162)]. The variancecovariance matrix

$\mathbf{Q}_{\mathrm{xxll}}=\left[\begin{array}{ll}\mathbf{Q}_{\mathrm{xx}} & \mathbf{Q}_{\mathrm{xl}} \\ \mathbf{Q}_{\mathrm{lx}} & \mathbf{Q}_{\mathrm{Il}}\end{array}\right]$

is essential, if further processing steps are intended, e.g. a rigorous combination of consecutive adjustment processes, cf. Sect. 8.

\section{Coordinate-based bundle adjustment}

The network adjustment is the first step of the processing. As a result, it provides the spatial coordinates and their corresponding uncertainties as the full variance-covariance matrix of the observed points. Depending on the extent of the local network and the accuracy requirements, the influence of the curvature of the Earth cannot be neglected. To overcome the influence of the inclination caused by the divergences of the plumb lines, different analysis strategies have been developed. Based on a spherical approximation, Schwarz (1994) suggests a geometric reduction to project the polar observations to a local Cartesian coordinate system. Another possibility is to introduce additional parameters that parametrize the influence of the plumb line direction [e.g. Heck (2003, chp. 4, pp. 82 ff.), Awange and Grafarend (2005, chp. 7, pp. 77 ff.)]. In metrology, coordinate-based algorithms have been developed [e.g. Calkins (2002), Lösler et al. (2015a)], because most of the instruments used in metrology and monitoring, e.g. Coordinate Measuring Machines and laser trackers, are unrelated to the gravity field. A detailed description of such an algorithm will be given in the next sections.

\subsection{Derivation of the functional model}

The process to combine several stand points or even instruments is based on homologous Cartesian coordinates. Each initialized stand point defines its own local true Cartesian coordinate system. Whereas a Coordinate Measuring Machine provides Cartesian coordinates, laser trackers and total stations provide polar measurements. The polar observations of the $i$ th point $\mathbf{p}$ of the $j$ th stand point have to be converted into Cartesian spatial coordinates 
$\mathbf{p}_{i, j}(\Theta, \Phi, d)=\left(\begin{array}{l}x \\ y \\ z\end{array}\right)_{i, j}=\left(\begin{array}{c}d \sin \Phi \cos \Theta \\ d \sin \Phi \sin \Theta \\ d \cos \Phi\end{array}\right)_{i, j}$.

Here, $d$ is the slope distance, and $\Theta$ and $\Phi$ are the yaw and pitch angles w.r.t. the local stand point coordinate system, respectively.

To combine the observed coordinates $\mathbf{p}_{i, j}$ of the $j$ th stand point with the ground-fixed global coordinate system $\mathbf{P}_{i}$, a conformal spatial parameters coordinate transformation can be used [cf. Shen et al. (2006), Lösler and Eschelbach (2014)], i.e.

$\mathbf{p}_{i, j}=\mathbf{T}_{j}+\mathbf{R}_{j} \mathbf{S}_{j} \mathbf{P}_{i}$

Here, the translation vector is denoted by $\mathbf{T}$, the diagonal scaling matrix is $\mathbf{S}$, which applies the scaling parameter $s$ uniformly to all axes by setting $\mathbf{S}=s \mathbf{E}$, and $\mathbf{R}$ is the rotation matrix. To estimate the global coordinates $\mathbf{P}_{i}$ and the unknown transformation parameters of each stand point, a Gauß-Markov model can be used [e.g. Mikhail and Ackerman (1976, chp. 5, pp. 101 ff.), Koch (1999, chp. 3, pp. 149 ff.)], expressed as

$\mathbf{A x}=\mathbf{l}+\mathbf{v}$

The design matrix $\mathbf{A}$, which contains the partial derivatives of the linearized functional model with respect to the unknown

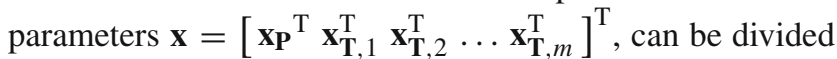
into a coordinate part $\mathbf{A}_{\mathbf{P}}$ and a part $\mathbf{A}_{\mathbf{T}}$, which contains the transformation parameters of the $m$ stand points. The local coordinates of each stand point are given by the (reduced) observation vector $\mathbf{l}$, and the vector $\mathbf{v}$ contains the observational residuals. The equation system, thus, becomes

$$
\begin{aligned}
& {\left[\begin{array}{ccccc}
\mathbf{A}_{\mathbf{P}, 1} & \mathbf{A}_{\mathbf{T}, 1} & \mathbf{0} & \cdots & \mathbf{0} \\
\mathbf{A}_{\mathbf{P}, 2} & \mathbf{0} & \mathbf{A}_{\mathbf{T}, 2} & & \vdots \\
\vdots & \vdots & & \ddots & \mathbf{0} \\
\mathbf{A}_{\mathbf{P}, m} & \mathbf{0} & \ldots & \mathbf{0} & \mathbf{A}_{\mathbf{T}, m}
\end{array}\right]\left[\begin{array}{c}
\mathbf{x}_{\mathbf{P}} \\
\mathbf{x}_{\mathbf{T}, 1} \\
\mathbf{x}_{\mathbf{T}, 2} \\
\vdots \\
\mathbf{x}_{\mathbf{T}, m}
\end{array}\right]} \\
& =\left[\begin{array}{c}
\mathbf{l}_{\mathbf{p}, 1} \\
\mathbf{l}_{\mathbf{p}, 2} \\
\vdots \\
\mathbf{l}_{\mathbf{p}, m}
\end{array}\right]+\left[\begin{array}{c}
\mathbf{v}_{\mathbf{p}, 1} \\
\mathbf{v}_{\mathbf{p}, 2} \\
\vdots \\
\mathbf{v}_{\mathbf{p}, m}
\end{array}\right] .
\end{aligned}
$$

The stochastic model of the local points is represented by $\mathbf{Q}_{\mathbf{p p}}$. Furthermore, prior results and/or GNSS observations $\overline{\mathbf{x}}_{\mathbf{P}}$ and their uncertainties $\mathbf{Q}_{\overline{\mathbf{x}}_{\mathbf{P}} \overline{\mathbf{x}}_{\mathbf{P}}}$ can be introduced to define the geodetic datum of the network by

$$
\left[\begin{array}{cc}
\mathbf{A}_{\mathbf{P}} & \mathbf{A}_{\mathbf{T}} \\
\mathbf{E} & \mathbf{0}
\end{array}\right]\left[\begin{array}{c}
\mathbf{x}_{\mathbf{P}} \\
\mathbf{x}_{\mathbf{T}}
\end{array}\right]=\left[\begin{array}{c}
\mathbf{l}_{\mathbf{p}} \\
\overline{\mathbf{x}}_{\mathbf{P}}
\end{array}\right]+\left[\begin{array}{c}
\mathbf{v}_{\mathbf{p}} \\
\mathbf{v}_{\overline{\mathbf{x}}_{\mathbf{P}}}
\end{array}\right]
$$

and the corresponding stochastic model reads

$\mathbf{Q}_{\mathrm{ll}}=\left[\begin{array}{cc}\mathbf{Q}_{\mathbf{p p}} & \mathbf{0} \\ \mathbf{0} & \mathbf{Q}_{\overline{\mathbf{x}}_{\mathbf{P}} \overline{\mathbf{x}}_{\mathbf{P}}}\end{array}\right]$

Additional restrictions $\mathbf{C}^{\mathrm{T}} \mathbf{x}=\mathbf{c}$ can be applied to constrain the number of transformation parameters, e.g. to fix the scale parameter to $s=1$, or to rectify the defect of the normal equation matrix in case of a free network adjustment

$\left[\begin{array}{cc}\mathbf{A}^{\mathrm{T}} \mathbf{Q}_{\mathbf{l l}}^{-\mathbf{1}} \mathbf{A} & \mathbf{C} \\ \mathbf{C}^{\mathrm{T}} & \mathbf{0}\end{array}\right]\left[\begin{array}{l}\mathbf{x} \\ \mathbf{k}\end{array}\right]=\left[\begin{array}{c}\mathbf{A}^{\mathrm{T}} \mathbf{Q}_{\mathbf{l l}}^{-\mathbf{1}} \mathbf{l} \\ \mathbf{c}\end{array}\right]$,

where $\mathbf{k}$ denotes the Lagrange multipliers. The adjustment model becomes bi-linear, if the rotation sequence $\mathbf{R}$ in Eq. 11 is parametrized by a quaternion. Thus, good convergence properties can be expected [cf. Lösler and Eschelbach (2012)].

\subsection{Assessment of the parameter selection for the functional model}

Applying the coordinate-based bundle adjustment as described in Sect. 4.1, each stand point will be optimally integrated into the global frame using up to seven parameters. This parametrization is also known as 6-DOF solution, because in general, the scale parameter is fixed to $s=1$. If the extent of the network is very small, the divergences of the plumb lines are often neglected, which means that the rotation angles $r_{x}$ and $r_{y}$ are fixed to $r_{x}=r_{y}=0$. Thus, the number of parameters per stand point is reduced from six to four (4-DOF).

In our case, the extent of the radome network does not exceed $25 \mathrm{~m}$. Increasing the number of parameters will reduce the squared sum of weighted residuals $\mathbf{v}^{\mathrm{T}} \mathbf{Q}_{\mathbf{l l}}^{-1} \mathbf{v}$, but it also increases the likelihood of an over-parametrization of the model. This problem is known as a model selection problem. To get an indication on which model should be used during the adjustment process, several approaches have been developed. Comprehensive textbooks on the topic of model selection are, e.g. Burnham and Anderson (2002) and Claeskens and Hjort (2008).

Based on information theory, one well-known method is the Akaike information criterion (AIC).

$\mathrm{AIC}=-2 \log \mathcal{L}\left(\mathbf{x}, \bar{\sigma}^{2} ; \mathbf{l}\right)+2(u+1)$

Here, $\mathbf{l}$ is the $n \times 1$ vector of observations, $\mathbf{x}$ is the $u \times 1$ vector of model parameters and $\bar{\sigma}^{2}$ is the variance factor that maximize the likelihood function $\mathcal{L}\left(\mathbf{x}, \bar{\sigma}^{2} ; \mathbf{l}\right)$, cf. Akaike (1974). The second term of the equation depends on the number of model parameters $u$ and can be interpreted as a penalty term. The preferable model is indicated by the smallest AIC value. 
Due to the penalty term the preferred model has an adequate number of parameters [e.g. Lehmann and Lösler (2016)].

Assuming observations with a normal distribution, the likelihood function reads [e.g. Koch (1999, chp. 3.2.4, p. 161)]

$\mathcal{L}\left(\mathbf{x}, \bar{\sigma}^{2} ; \mathbf{l}\right)=\frac{1}{\left(2 \pi \bar{\sigma}^{2}\right)^{n / 2}} \exp \left(-\frac{\mathbf{v}^{\mathrm{T}} \mathbf{Q}_{\mathbf{l l}}^{-1} \mathbf{v}}{2 \bar{\sigma}^{2}}\right)$

which is equivalent to

$\log \mathcal{L}\left(\mathbf{x}, \bar{\sigma}^{2} ; \mathbf{l}\right)=-\frac{n}{2} \log (2 \pi)-\frac{n}{2} \log \bar{\sigma}^{2}-\frac{\mathbf{v}^{\mathrm{T}} \mathbf{Q}_{\mathbf{~ I l}}^{-1} \mathbf{v}}{2 \bar{\sigma}^{2}}$.

The maximum likelihood estimator $\bar{\sigma}^{2}$ of the unknown variance factor $\sigma^{2}$ [e.g. Koch (1999, chp. 3.2.4, p. 162)] is given by

$\bar{\sigma}^{2}=\frac{\mathbf{v}^{\mathrm{T}} \mathbf{Q}_{\mathbf{l l}}^{-1} \mathbf{v}}{n}$,

and the likelihood function in Eq. 17 follows by substituting Eq. 20 in Eq. 19 and becomes

$\log \mathcal{L}\left(\mathbf{x}, \bar{\sigma}^{2} ; \mathbf{l}\right)=-\frac{n}{2} \log (2 \pi)-\frac{n}{2} \log \frac{\mathbf{v}^{\mathrm{T}} \mathbf{Q}_{\mathbf{l l}}^{-1} \mathbf{v}}{n}-\frac{n}{2}$.

AIC may fail, if the number of observations $n$ is small w.r.t. the number of parameters $u$. Thus, the usage of a secondorder improved AIC is strongly recommended [e.g. Burnham and Anderson (2002, chp. 7.4, pp. $374 \mathrm{ff}$.)]

$\mathrm{AIC}_{c}=\mathrm{AIC}+\frac{2(u+1)(u+2)}{n-u-2}$.

Because $\mathrm{AIC}_{c}$ tends to AIC if $n$ gets large, $\mathrm{AIC}_{c}$ should always be used. There are alternative criteria to select the preferable model but they only differ in the limit range [cf. Lehmann (2014)].

\subsection{Stochastic model for polar measurements instruments}

The stochastic model describes the a priori uncertainties of the measurement process and allows for combining different types of observations w.r.t. their uncertainties. In general, the uncertainties are composed of various factors and statistical distributions [e.g. Adunga (2007, chp. 5, pp. 93 ff.), DIN 1319 (1995), GUM (2008a, b)]. that:

Equation 10 assumes a perfect instrument which means

- the yaw and pitch axes are orthogonal to each other and have an intersection point,
- the distance measurement unit is centered w.r.t. this intersection point,

- the target beam is coaxial and orthogonal to the elevation axis,

- the normal vector of the angle encoder is aligned and centered to the rotation axis, and

- the angle encoders are free of graduation errors.

Due to manufacturing reasons, deviations from the idealized model are possible, cf. Fig. 3. Ideally, the largest share of these errors is compensated by the manufacturer's firmware, and only a few errors can be, and need to be, rechecked by the instrument operator. Based on the work of Muralikrishnan (2009), Hughes et al. (2011) suggest a compensation model for a mobile laser tracker, which is also valid for other polar measurement instruments, e.g. total stations or laser scanners.

The corrected slope distance $\hat{d}$ is calculated by adding a displacement offset $\lambda$ and a distance-dependent scaling factor $\mu$, i.e.

$\hat{d}=(1+\mu) d+\lambda$.

The angle encoder errors are parametrized as Fourier series:

$$
\begin{aligned}
& \hat{\Theta}=\Theta+a_{\Theta, 0}+\sum_{q=1}^{n_{q}}\left(a_{\Theta, q} \cos q \Theta+b_{\Theta, q} \sin q \Theta\right), \\
& \hat{\Phi}=\Phi+a_{\Phi, 0}+\sum_{q=1}^{n_{q}}\left(a_{\Phi, q} \cos q \Phi+b_{\Phi, q} \sin q \Phi\right) .
\end{aligned}
$$

Here, $a_{q}$ and $b_{q}$ represent the Fourier coefficients and $n_{q}$ symbolizes the harmonic order of the Fourier series. Laboratory investigation shows that it is sufficient to restrict the harmonic order to $n_{q}=2$ (Lewis at al. 2011). The coefficient $a_{\Theta, 0}$ describes an angular zero offset of the yaw angle encoder. In general, a rectified orientation parameter is introduced during the network adjustment so that $a_{\Theta, 0}$ can instead be set to zero.

The functional relation between the polar observations and the Cartesian coordinates can be expressed as

$\mathbf{p}_{i, j}(\hat{\Theta}, \hat{\Phi}, \hat{d})=\mathbf{p}_{0}+\mathbf{b}(\hat{\Theta}, \hat{\Phi})+\hat{d} \mathbf{n}(\hat{\Theta}, \hat{\Phi})$,

with

$$
\begin{aligned}
\mathbf{b}(\hat{\Theta}, \hat{\Phi})= & \mathbf{R}_{\hat{\Theta}}^{z}\left(\begin{array}{c}
e_{\Theta, \Phi} \\
0 \\
0
\end{array}\right) \\
& +\mathbf{R}_{\hat{\Theta}}^{z} \mathbf{R}_{\kappa}^{x} \mathbf{R}_{\hat{\Phi}-\frac{\pi}{2}}^{y} \mathbf{R}_{-\kappa}^{x}\left(\begin{array}{c}
t_{d, x}-e_{\Theta, \Phi} \\
t_{d, y} \\
t_{d, z}
\end{array}\right)
\end{aligned}
$$


Fig. 3 Schematic representation of deviations of a polar measurement instrument, e.g. a total station or a mobile laser tracker, proposed by Hughes et al. (2011). See text for further explanations

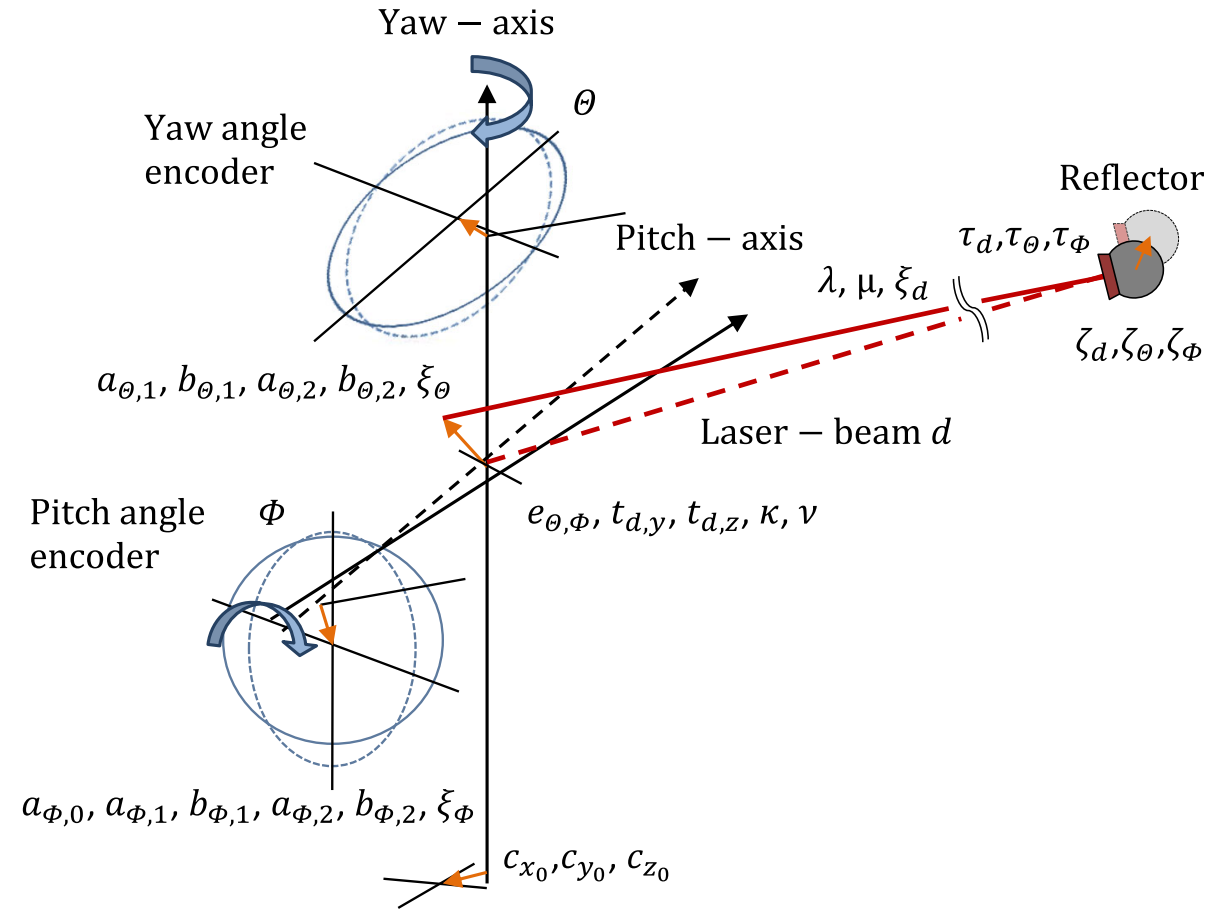

and

$\mathbf{n}(\hat{\Theta}, \hat{\Phi})=\mathbf{R}_{\hat{\Theta}}^{z} \mathbf{R}_{\kappa}^{x} \mathbf{R}_{\hat{\Phi}-\frac{\pi}{2}}^{y} \mathbf{R}_{-\kappa}^{x} \mathbf{R}_{\nu}^{z}\left(\begin{array}{l}1 \\ 0 \\ 0\end{array}\right)$.

A detailed derivation of Eqs. 26, 27 and 28 is given in the Appendix.

The coordinates of the stand point are summarized in the vector $\mathbf{p}_{0}$, the vector $\mathbf{b}$ considers the axis-offset $e_{\Theta, \Phi}$ and the centering error of the distance measurement unit $\left(t_{d, x} t_{d, y} t_{d, z}\right)^{\mathrm{T}}$. The vector $\mathbf{n}$ compensates for the trunnion axis error $\kappa$ and the horizontal collimation error $\nu$. The displacement offset $\lambda$ and $t_{d, x}$ are aligned to each other, so both are inseparable. Thus, $\lambda$ compensates for the misalignment of the distance measurement unit by constraining $t_{d, x}=0$. Even if this model was derived for laser trackers [e.g. Lösler et al. (2015b)], it is valid for total stations, too.

Equation 26 shows the geometrically related factors of the instrument, which are equivalent for all registered observations. In addition to the geometrically related factors, each measurement can be considered as a realization of a random experiment. Expanding Eqs. 23, 24 and 25 by a target centering error $\zeta$, a resolution limiting error of the digital output $\xi$, and a random error $\tau$ of the individual measurement process, the functional relations of the polar observations become

$\hat{d}=\left(1+\mu+\tau_{d}\right) d+\lambda+\zeta_{d}+\xi_{d}$,

$$
\begin{aligned}
\hat{\Theta}= & \Theta+\tau_{\Theta}+\xi_{\Theta}+\frac{\zeta_{\Theta}}{d} \rho+a_{\Theta, 0} \\
& +\sum_{q=1}^{n_{q}}\left(a_{\Theta, q} \cos q \Theta+b_{\Theta, q} \sin q \Theta\right), \\
\hat{\Phi}= & \Phi+\tau_{\Phi}+\xi_{\Phi}+\frac{\zeta_{\Phi}}{d} \rho+a_{\Phi, 0} \\
& +\sum_{q=1}^{n_{q}}\left(a_{\Phi, q} \cos q \Phi+b_{\Phi, q} \sin q \Phi\right),
\end{aligned}
$$

where $\rho=\frac{\pi}{200 \text { gon }}$ denotes the angle conversion factor between radian and gon that transforms a small metric value into its angular representation using its distance $d$.

Furthermore, the centering error of the instrument $\mathbf{Q}_{\mathbf{p}_{0}}$ has to be taken into account if forced centering is used (Lösler and Eschelbach 2012). Applying the law of uncertainty propagation to Eq. 26, including Eqs. 29, 30 and 31, provides the a priori variance-covariance matrix $\mathbf{Q}_{\mathbf{p p}}$ of statically observed coordinates $\mathbf{p}$. Table 1 summarizes the parameters of the comprehensive stochastic model that we derived for the analysis of the polar measurements.

If glass body reflectors are used, it is important to align the normal of the reflector surface to the line of sight, to avoid systematic lateral $\varepsilon_{\text {lateral }}$ and radial $\varepsilon_{\text {radial }}$ errors [e.g. Pauli (1969), Rüeger (1996, chp. 10.2.5, pp. 158 ff.)]. The magnitude of the errors caused by a misaligned reflector depends on the reflector type and on the angle of incidence $\delta$ :

$$
\varepsilon_{\text {radial }}=d\left(n_{r}-\sqrt{n_{r}^{2}-\sin ^{2} \delta}\right)-e(1-\cos \delta),
$$


Table 1 Parameters of the comprehensive stochastic model for the least-squares adjustment of polar measurements

\begin{tabular}{|c|c|c|}
\hline Parameter name & Abbreviation & unit \\
\hline \multirow[t]{3}{*}{ Centering error } & $c_{x_{0}}$ & {$[\mathrm{~m}]$} \\
\hline & $c_{y_{0}}$ & {$[\mathrm{~m}]$} \\
\hline & $c_{z_{0}}$ & {$[\mathrm{~m}]$} \\
\hline EDM scale error & $\mu$ & [ppm \\
\hline EDM displacement offset & $\lambda$ & {$[\mathrm{m}]$} \\
\hline \multirow{4}{*}{ Yaw angle encoder $q=2$} & $a_{\Theta, 1}$ & [gon] \\
\hline & $a_{\Theta, 2}$ & [gon] \\
\hline & $b_{\Theta, 1}$ & [gon] \\
\hline & $b_{\Theta, 2}$ & [gon] \\
\hline \multirow[t]{5}{*}{ Pitch angle encoder $q=2$} & $a_{\Phi, 0}$ & [gon] \\
\hline & $a_{\Phi, 1}$ & [gon] \\
\hline & $a_{\Phi, 2}$ & [gon] \\
\hline & $b_{\Phi, 1}$ & [gon] \\
\hline & $b_{\Phi, 2}$ & [gon] \\
\hline Trunnion axis error & $\kappa$ & [gon] \\
\hline Horizontal collimation error & $v$ & [gon] \\
\hline Laser beam/axis offset & $e_{\Theta, \Phi}$ & {$[\mathrm{m}]$} \\
\hline \multirow[t]{2}{*}{ Laser beam offset } & $t_{d, y}$ & {$[\mathrm{~m}]$} \\
\hline & $t_{d, z}$ & {$[\mathrm{~m}]$} \\
\hline \multirow[t]{3}{*}{ Target centering error } & $\zeta_{d}$ & {$[\mathrm{~m}]$} \\
\hline & $\zeta_{\Theta}$ & {$[\mathrm{m}]$} \\
\hline & $\zeta_{\Phi}$ & {$[\mathrm{m}]$} \\
\hline \multirow[t]{3}{*}{ Resolution error } & $\xi d$ & {$[\mathrm{~m}]$} \\
\hline & $\xi \Theta$ & [gon] \\
\hline & $\xi \Phi$ & [gon] \\
\hline \multirow[t]{3}{*}{ Measurement noise } & $\tau_{d}$ & {$[\mathrm{~m}]$} \\
\hline & $\tau_{\Theta}$ & [gon] \\
\hline & $\tau_{\Phi}$ & [gon] \\
\hline
\end{tabular}

$\varepsilon_{\text {lateral }}=(d-e) \sin \delta-d \sec \delta_{G} \sin \left(\delta-\delta_{G}\right)$.

Here, $\delta_{G}=\arcsin \frac{\sin \delta}{n_{r}}, e$ denotes the distance between the front surface of the prism and the center-symmetric point, and $d$ denotes the distance between the front surface of the prism and the corner point of the triple prism. The ratio of the group refractive indices of glass and air is given by $n_{r} \approx 1.52$ [cf. Rüeger (1996, p. 155)].

Figure 4 depicts the resulting systematic errors caused by the angle of incidence $\delta$ for different reflector types. It is shown, that small size glass body reflectors yield a lower error level. On the other hand, these reflectors reflect only a small part of the instrument's laser beam and the small usable laser spot size increases the likelihood for measurement failure. The likelihood of measurement failure is even higher if the reflector is misaligned.

Misalignment occurs often if standard reflectors are observed that are mounted at the turnable part of the radio
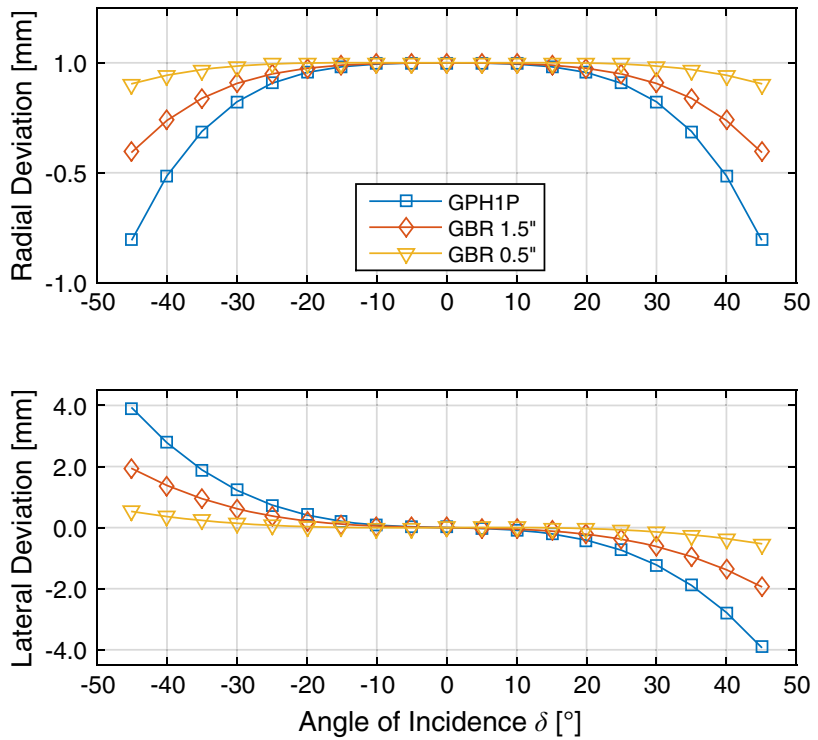

Fig. 4 Radial and lateral deviations caused by reflector misalignment for precision reflector GPH1P, glass ball reflector GBR1.5", and glass ball reflector GBR0.5"

telescope. In this case, the angle of incidence $\delta$ depends on the radio telescope orientation. If the normal of the reflector surface is known in a reference position of the radio telescope, these systematic errors can be corrected for all radio telescope orientations (Lösler et al. 2013). The remaining residual uncertainty is similar to the centering error $\zeta$ and can be taken into account in the network adjustment process (Lösler et al. 2015a).

\subsection{Time-dependent correlations of the observations}

Up to here the measurement process of a single point was described and the uncertainties of a statically observed position were derived. Besides the random part of the observation process a time-dependent or so-called signal part is recommended, and both are assumed to be uncorrelated with zero means [e.g. Mikhail and Ackerman (1976, p. 399), Kuhlmann (2003)]. Thus, the resulting variance-covariance matrix becomes

$\mathbf{Q}_{\mathrm{II}}=\mathbf{Q}_{\mathrm{s}}+\mathbf{Q}_{\mathrm{pp}}$

In most cases, the uncertainties $\mathbf{Q}_{\mathbf{p p}}$ of the measurement process are known, e.g. by specification of the manufacturer, or can be realistically assessed, e.g. by calibration reports or practical knowledge, and introduced during the data analysis, e.g. the network adjustment. However, statistical temporal dependencies between the observations are neglected and $\mathbf{Q}_{\mathrm{s}} \approx \mathbf{0}$ is implied. If high-frequent measurements are carried out during a monitoring process, the so-called persistence of a series, which describes the temporal or spatial dependency of 
Fig. 5 Examples of typical auto-covariance functions analyzed by Taubenheim (1969, chp. 7.4, pp. 227 ff.), Bähr and Richter (1975)

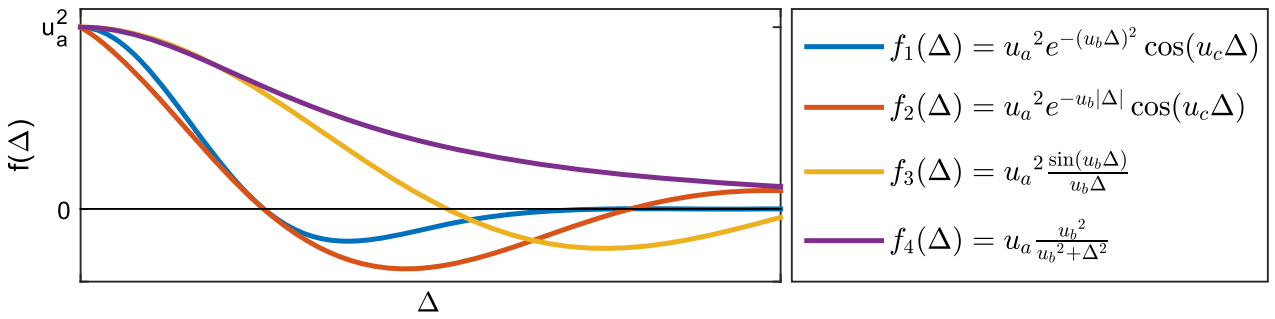

the observations (Taubenheim 1969, p. 17), should be taken into account. The measure of the persistence of the signal can be expressed by the auto-covariance function. For a given discrete series of length $n$ the empirical auto-covariance function is

$f(\Delta)=\frac{1}{n-\Delta-1} \sum_{t}^{n-\Delta}\left(l_{t}-\hat{l}\right)\left(l_{t+\Delta}-\hat{l}\right)$,

where $l_{t}$ is an observed value of an equidistant sample $\mathbf{l}$ and $\hat{l}$ is the average of the sample. To avoid an overestimation, the order of overlapping should be restricted to $\Delta=\frac{n}{10}$ [cf. Heunecke et al. (2013, p. 343)].

In practical application it is, in general, not economical to use empirical covariance functions. Therefore, theoretically determined covariance functions are derived from a given data sample by a least-squares adjustment and transferred to the actual measuring process [e.g. Mikhail and Ackerman (1976, chp. 14, pp. 393 ff.), Heunecke et al. (2013, chp. 8.4.2, pp. 317 ff.)]. Suitable functions are discussed by, e.g. Taubenheim (1969, chp. 7.4, pp. 227 ff.), Bähr and Richter (1975), and presented in Fig. 5.

The selected and determined covariance function can be used as an appropriated approximation of the true covariance function to derive a theoretical variance-covariance matrix $\mathbf{Q}_{\text {s }}$ of the signal.

\section{Latency time of polar observations}

The observations $d, \Theta$ and $\Phi$ of the polar measurement instrument relate to different points of time. Depending on the velocity of the object, latency becomes significant and has to be taken into account. Whereas the corresponding yaw and pitch angles can be assumed to be observed synchronously, the distance measurement has a time difference with respect to the angular measurements [e.g. Krickel (2004), Stempfhuber (2004)].

The magnitude of this time difference depends on the particular type of instrument that is used. Modern instruments trigger the angle encoder with up to $5 \mathrm{kHz}$, to achieve an accurate time mapping [e.g. Lienhart et al. (2009)] and an improved automatic target recognition will reduce the centering errors of measurements in motion [e.g. Grimm and Hor- nung (2015)]. Moreover, the so-called wave-form-digitizing technique of the electro-optical distance measurement unit of the used MS50 (Leica) is two times faster than the common phase-shift measurement systems, which reduces the recording time [cf. Grimm (2014)].

During the regular observation process of a VLBI session, the radio telescope compensates for the Earth's rotation while a radio source is tracked, see Sect. 1. The tracking velocities of the telescope are sufficiently slow to observe targets that are mounted on the radio telescope with a total station. If the latency time $\delta t_{\text {lat }}$ and the corresponding uncertainty $u_{\delta t_{\text {lat }}}$ are known, the resulting uncertainties can be estimated by Eq. 1. The coordinates of $\tilde{\mathbf{P}}_{i, t_{i}}$ and $\tilde{\mathbf{P}}_{i, t_{i}-\delta t_{\text {lat }}}$ denote the true positions at the time of measurement $t_{i}$ and the position w.r.t. the delay, respectively. Inverting Eq. 10, the polar observations $\Theta_{i, t_{i}}, \Phi_{i, t_{i}}$ and $d_{i, t_{i}}$ as well as $\Theta_{i, t_{i}-\delta t_{\text {lat }}}, \Phi_{i, t_{i}-\delta t_{\text {lat }}}$ and $d_{i, t_{i}-\delta t_{\text {lat }}}$ can be derived and recombined. Under the assumption that the angular measurements are related to $t_{i}$ and the distance measurement is asynchronous, the observed position $\mathbf{P}_{i, t_{i}}$ results from $\Theta_{i, t_{i}}, \Phi_{i, t_{i}}$ and $d_{i, t_{i}-\delta t_{\mathrm{lat}}}$. Therefore, the error of the latency $\boldsymbol{\varepsilon}_{\text {lat }}=\mathbf{P}_{i, t_{i}}-\tilde{\mathbf{P}}_{i, t_{i}}$ could be corrected, if $\delta t_{\text {lat }}$ is known. To derive the uncertainties caused by the latency time, a Monte Carlo simulation can be applied. For this purpose, the latency time $\delta t_{\text {lat }}$ is simulated $m=100,000$ times w.r.t. $u_{\delta t_{\text {lat }}}$. The $m \times 1$ residual vectors $\boldsymbol{\varepsilon}_{x}, \boldsymbol{\varepsilon}_{y}, \boldsymbol{\varepsilon}_{z}$ provide the $3 \times 3$ variance-covariance matrix $\mathbf{Q}_{\text {lat }, i}$ of the $i$ th position, i.e.

$\mathbf{Q}_{\text {lat }, i}=\frac{1}{m}\left(\begin{array}{ccc}\boldsymbol{\varepsilon}_{x}^{\mathrm{T}} \boldsymbol{\varepsilon}_{x} & \boldsymbol{\varepsilon}_{x}^{\mathrm{T}} \boldsymbol{\varepsilon}_{y} & \boldsymbol{\varepsilon}_{x}^{\mathrm{T}} \boldsymbol{\varepsilon}_{z} \\ & \boldsymbol{\varepsilon}_{y}^{\mathrm{T}} \boldsymbol{\varepsilon}_{y} & \boldsymbol{\varepsilon}_{y}^{\mathrm{T}} \boldsymbol{\varepsilon}_{z} \\ \text { symm. } & & \boldsymbol{\varepsilon}_{z}^{\mathrm{T}} \boldsymbol{\varepsilon}_{z}\end{array}\right)$.

Due to various directions of movement of the radio telescope, the sub-matrices $\mathbf{Q}_{\text {lat }, i}$ have to be derived individually for each measured position. Each matrix is unique and reflects the geometric configuration at the time of observation.

\section{Radio telescope angle encoders}

In addition to the estimated coordinates, the radio telescope angles $\alpha$ and $\epsilon$ are used as observations to solve Eq. 1. Thus, the uncertainties of both, the azimuth- and the 
elevation-angle encoder, have to be taken into account. In most cases, the uncertainties of the radio telescope angles are assumed to be uncorrelated and only a diagonal variance matrix is introduced to the least-squares adjustment [e.g. Lösler (2008), Kallio and Poutanen (2012), Ning et al. (2015)], but in principle, the construction of an azimuthelevation type radio telescope is similar to a total station as described in Sect. 4. Consequently, the same considerations can be applied. Irregularities in the construction of the radio telescope like the axis-offset are formulated as unknown parameters in Eq. 1 and will be estimated within the least-squares adjustment. Therefore, the angle-encoder uncertainties can be derived by Eqs. 30 and 31, and provide the fully populated variance-covariance matrices $\mathbf{Q}_{\alpha}$ and $\mathbf{Q}_{\epsilon}$. Besides a random measurement noise, $\mathbf{Q}_{\alpha}$ and $\mathbf{Q}_{\epsilon}$ take the resolution of the encoder as well as the production-related uncertainties into account.

\section{Synchronization error}

The combination of several sensors requires a timeline of observations in a consistent temporal reference frame. The demands on the quality of the synchronization vary. In most cases, the environmental temperature, that is used for compensating errors of the distance measurement unit of the total station [e.g. Ciddor (2002)], is not time critical. However, the synchronization of the total station and the radio telescope is important for the measurement process and for the data analysis, in particular if the data are synchronized and analyzed in post-processing.

Being $\delta t_{\text {syn }}$ the synchronization error between the total station and the radio telescope and $t_{i}$ the reference time of measurement, the uncertainties can be assumed to have a continuous uniform distribution. Thus, each radio telescope angle has equal probability within the interval $\pm \delta t_{\text {syn }}$. The variances of the azimuth and elevation angles, respectively, caused by a synchronization error are [e.g. Adunka (2007, chp. 4.2.1, pp. 66-67), GUM (2008a, p. 13)]

$u_{\alpha_{i}, \delta t_{\mathrm{syn}}}^{2}=\frac{1}{12}\left(\alpha_{t_{i}+\delta t_{\mathrm{syn}}}-\alpha_{t_{i}-\delta t_{\mathrm{syn}}}\right)^{2}$

and

$u_{\epsilon_{i}, \delta t_{\mathrm{syn}}}^{2}=\frac{1}{12}\left(\epsilon_{t_{i}+\delta t_{\mathrm{syn}}}-\epsilon_{t_{i}-\delta t_{\mathrm{syn}}}\right)^{2}$

The azimuth angle $\alpha_{t_{i}}$ and the elevation angle $\epsilon_{t_{i}}$ as well as the radio telescope's direction of motion depend on the currently observed radio source position, thus, the magnitude of the uncertainty is changing w.r.t. the observed radio source. It should be noted that the synchronization error is not an angle encoder error of the radio telescope. Similar to the uncertainties caused by the latency time in Sect. 5, the influence of $\delta t_{\text {syn }}$ can be derived using Eq. 1, but needs higher computational effort.

\section{Recursive parameter estimation}

If observations $\mathbf{I}$ are collected gradually, a recursive parameter estimation, i.e. a special case of a Kalman (1960) filter process, can be used to gradually improve the unknown parameters $\hat{\mathbf{x}}$. Instead of Eq. 14, an improvement $\delta \mathbf{x}_{k-1, k}$ of the current state-vector $\hat{\mathbf{x}}_{k-1}$ can be derived by [e.g. Koch (2007, chp. 4.2.7, pp. 107 ff.), Teunissen (2009, chp. 2.3, pp. 54 ff.)]

$\hat{\mathbf{x}}_{k}=\hat{\mathbf{x}}_{k-1}+\delta \mathbf{x}_{k-1, k}=\hat{\mathbf{x}}_{k-1}+\mathbf{K}_{k-1, k}\left(\mathbf{l}_{k}-\mathbf{A} \hat{\mathbf{x}}_{k-1}\right)$.

Here $\mathbf{A}$ is the coefficient matrix that contains the partial derivations w.r.t. the unknown parameters, and $\mathbf{K}$ is the gain matrix

$\mathbf{K}_{k-1, k}=\mathbf{Q}_{\hat{\mathbf{x}} \hat{\mathbf{x}}_{k-1}} \mathbf{A}^{\mathrm{T}}\left(\mathbf{Q}_{\mathbf{l} k}+\mathbf{A} \mathbf{Q}_{\hat{\mathbf{x}} \hat{\mathbf{x}}_{k-1}} \mathbf{A}^{\mathrm{T}}\right)^{-1}$

The matrix $\mathbf{Q}_{\mathbf{l}}{ }_{k}$ is the variance-covariance matrix of the additional observations $\mathbf{l}_{k}$ of the $k$ th state. The corresponding uncertainties of the unknown parameters of the $k$ th state vector is given by

$\mathbf{Q}_{\hat{\mathbf{x}} \hat{\mathbf{x}}_{k}}=\mathbf{Q}_{\hat{\mathbf{x}} \hat{\mathbf{x}}_{k-1}}-\mathbf{K A} \mathbf{Q}_{\hat{\mathbf{x}} \hat{\mathbf{x}}_{k-1}}$

To combine the results of single adjustment processes of several epochs, Eqs. 39-41 can be simplified to the case where the parameters are observed directly. Setting $\mathbf{A}=\mathbf{E}$, $\mathbf{l}_{k}=\mathbf{x}_{k}$ and $\mathbf{Q}_{\mathbf{l l}_{k}}=\mathbf{Q}_{\mathbf{x x} k}$, where $\mathbf{x}_{k}$ denotes the solution of the $k$ th monitoring epoch that contains the reference point and additional radio telescope parameters as well as the coordinates of the marked points of the network, and $\mathbf{Q}_{\mathbf{x x} k}$ is the corresponding variance-covariance matrix derived by Eqs. 9, 39-41 can be used to combine the single solutions.

\section{Monitoring during CONT14}

During the global 15-day-long very long baseline interferometry (VLBI) campaign CONT14 in May 2014, a total station MS50 (Leica) was used to monitor the IVS reference point of the $20 \mathrm{~m}$ radio telescope at the Onsala Space Observatory during the ongoing telescope operations. The $20 \mathrm{~m}$ azimuthelevation type radio telescope is enclosed by a protection radome. The network inside the radome is realized by five stable survey pillars on the radome foundation wall. In addition to these survey pillars, there are three survey markers with center holes in the ground of the radome building, and three 


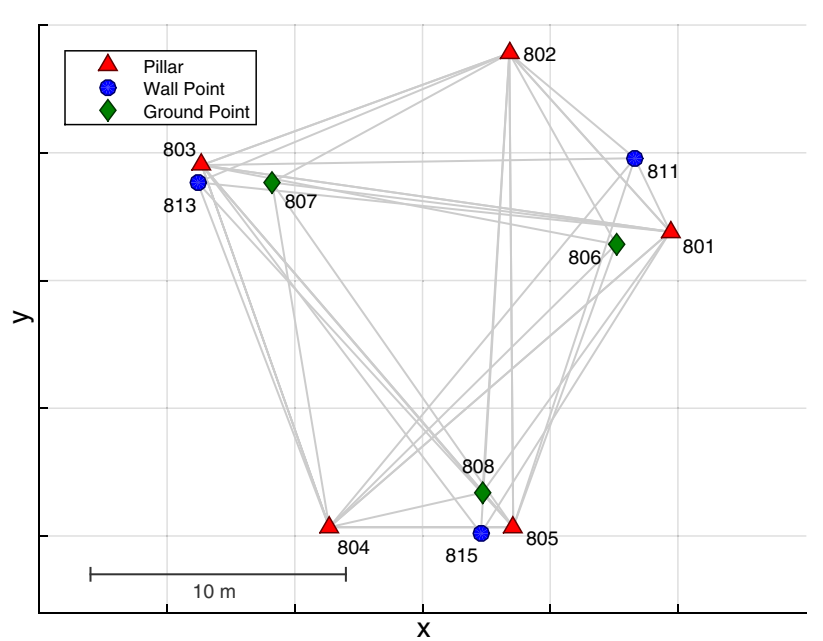

Fig. 6 Survey network inside the radome of the $20 \mathrm{~m}$ radio telescope at the Onsala Space Observatory with observable connections w.r.t. the survey pillars

additional magnetic reflector holders that support $1.5^{\prime \prime}$ reflectors are mounted at the radome foundation wall and allow for a combination of the radome network with the local site network outside the building. To monitor the reference point during CONT14, only the radome network was involved.

Figure 6 depicts the radome network and the observable connections when the pillars are used as instrument stand points. To carry out the indirect determination of the reference point, six 1.5" spherical reflectors $\left(\mathrm{GBR} 1.5^{\prime \prime}\right)$ with glass bodies and six Leica GMP104 prisms were mounted at the elevation cabin and at the counterweight of the radio telescope. The total station was placed on the survey pillars and initialization measurements were preformed. To check the setup of the current stand point, measurements to the remaining points of the radome network were carried out continually during the monitoring process. Due to the continuously (slightly) moving radio telescope during the VLBI campaign CONT14, see Sect. 1, all measurements were carried out in one face only in a rapid measurement mode. Thus, the total station was calibrated right in the beginning of the monitoring, following the calibration procedure that is proposed by the manufacturer using an overdetermined setup.

From a single observation stand point all visible reflectors on the radio telescope were observed with the total station once and in one face while the telescope was tracking a radio source. These observations formed one measurement set. Which reflector would be visible for each measurement set was calculated beforehand in HEIMDALL, based on a priori information on the position of the total station, the position of the reflectors on the radio telescope, and the tracking orientation of the telescope. Reflectors with unfavorable incident angles were not included in the measurement sets. On average for about $79 \%$ of the telescope tracking positions, reflectors were visible and could be measured. The number of reflectors that could be measured in a measurement set also depended on the time that the radio telescope was tracking a radio source. The minimum and maximum number of reflectors measured in a measurement set were 1 and 7, respectively, while the average number was 3 . During the fast slewing movements when the radio telescope changed its orientation from one radio source to another, no total station measurements to reflectors on the radio telescope were performed.

A one-sided point distribution of telescope points negatively effects the estimated position of the reference point [e.g. Lösler et al. (2013), Lossin et al. (2014)]. Therefore, at least two observation stand points in (nearly) diametrically opposed order were used per monitoring experiment, but in most cases the number of stand points was larger than two and an additional scaling parameter as suggested by Lösler et al. (2013) could be neglected. Table 3 summarizes the network configuration, the number of pillars used as instrument stand points, the total number of points that were observed at the radio telescope during the monitoring epoch, and the degree of freedom (DOF) of the coordinate-based bundle adjustment. The observation time per stand point varied between $3.5,6$ and $7 \mathrm{~h}$ for configurations using five, three and two pillars as instrument stand points, respectively, excluding the setup time of about $1 \mathrm{~h}$ per stand point and maintenance time between the epochs. In total 15 monitoring epochs were carried out during the ongoing CONT14 VLBI campaign.

In comparison to the number of observed points at the radio telescope, the degree of freedom is small. The reason is that each point at the radio telescope could only be observed once. Thus, the degree of freedom of the bundle adjustment results entirely from redundant observations to the control points of the radome network. The estimated variance factor of the adjustment process can only be derived from the redundant part of the network. In most cases, this value will be too optimistic because of an incomplete stochastic model, an unrepresentative sample of the population, and the sample size [cf. Hennes (2007), Xu (2013)]. The transferability of the estimated variance factor to the non-redundant points at the radio telescope seems thus to be questionable and was, therefore, discarded.

To validate the number of parameters that are estimated during the network adjustment w.r.t. the rotation parameters $r_{x}$ and $r_{y}$, the $\mathrm{AIC}_{c}$ was used, see Sect. 4.2. For this purpose, each network solution was evaluated twice, once with the 4DOF approach and once with the 6-DOF approach. Whereas the 4-DOF solution restricts the number of parameters per stand point by fixing the rotation parameters $r_{x}=r_{y}=0$, the 6-DOF solution contains the rotation parameters $r_{x}$ and $r_{y}$ as additional parameters. As pointed out, the observed points at the operating radio telescope cannot be checked redundantly. A single polar measurement, which consists of the slope distance, the yaw angle and the pitch angle, increases 
Table 2 Comparison of the estimated $\mathrm{AIC}_{c}$ for each coordinate-based bundle adjustment, w.r.t. the 4-DOF and 6-DOF solution, respectively

\begin{tabular}{llllll}
\hline DOY & $\mathrm{AIC}_{c}$ & & & \multicolumn{2}{c}{ Preferred model } \\
\cline { 6 - 7 } \cline { 5 - 6 }$(2014)$ & 4 -DOF & 6 6-DOF & & 4 -DOF & 6 -DOF \\
\hline 126 & 364.2 & 330.8 & $\circ$ & $\checkmark$ \\
127 & 429.7 & 440.2 & $\checkmark$ & $\circ$ \\
128 & 467.8 & 398.1 & $\circ$ & $\checkmark$ \\
129 & 410.3 & 236.0 & & $\circ$ & $\checkmark$ \\
130 & 395.3 & 378.8 & & $\checkmark$ & $\checkmark$ \\
131 & 438.1 & 351.3 & & $\checkmark$ \\
132 & 506.4 & 417.3 & $\circ$ & $\checkmark$ \\
133 & 456.1 & 441.6 & $\circ$ & $\checkmark$ \\
134 & 257.4 & 292.9 & $\checkmark$ & $\circ$ \\
135 & 322.1 & 113.9 & $\circ$ & $\checkmark$ \\
136 & 458.7 & 432.7 & $\circ$ & $\checkmark$ \\
137 & 495.4 & 304.1 & $\circ$ & $\checkmark$ \\
138 & 410.7 & 158.8 & $\circ$ & $\checkmark$ \\
139 & 326.6 & 67.4 & $\circ$ & $\checkmark$ \\
140 & 213.6 & 178.0 & $\circ$ & $\checkmark$
\end{tabular}

The preferable model is indicated by the smaller $\mathrm{AIC}_{c}$ value. The checkmarks in the last two columns show whether the preferred model is the 4-DOF or the 6-DOF solution

the number of observations $n$ and unknowns $u$ by three, respectively. Thus, the degree of freedom does not change and these observations do not participate in the squared sum of weighted residuals $\mathbf{v}^{\mathrm{T}} \mathbf{Q}_{\mathrm{II}}^{-1} \mathbf{v}$. In Eq. 20 the maximum likelihood estimator of the variance factor is biased and decreases while $n$ increases. To reduce the influence of the biased $\bar{\sigma}^{2}$, the non-redundant points were excluded during the estimation process of $\mathrm{AIC}_{c}$. Table 2 shows the $\mathrm{AIC}_{c}$ for the 4-DOF and 6-DOF solution, respectively. The check-marks in the last two columns show whether the preferred model is the 4-DOF or the 6-DOF solution. For 13 out of 15 bundle adjustments the 6-DOF solution is preferred. Thus, to achieve consistency in the adjustment process, we chose to use the 6-DOF approach for all epochs.

After CONT14 we carried out the measurements for determining the time-dependent correlations of the total station observations. A reflector was mounted at the fixed construction near the elevation cabin of the radio telescope and observed with the total station during a period of $48 \mathrm{~h}$ and with a measurement frequency of every $30 \mathrm{~s}$. During the regular monitoring process, the total station aligns in the direction towards the predicted position of the target, carries out the automated target recognition and starts the measurement. The same procedure, consisting of aligning, targeting and measuring was chosen for the continuous measurements to the fixed installed reflector to take into account possible swinging characteristics of the compensator. The recorded data series was used to derive the temporal dependency of the obser- vations as described in Sect. 4.4 and introduced to the data preparation process, cf. Sect. 10.

\section{Data preparation for reference point determination}

To derive coordinates $\mathbf{P}$ and their uncertainties $\mathbf{Q P P}$ in a consistent reference frame, a network adjustment is needed. In contrast to the concept presented by Lösler et al. (2013), the network adjustment module was replaced by the coordinatebased algorithm described in Sect. 4. The 15 monitoring epochs were adjusted separately in a free network adjustment, cf. Eq. 16. The geodetic datum was realized by the ground markers and the pillars.

Initially, a GNSS campaign was carried out outside the radome to estimate global Cartesian coordinates of a part of the local site network using the precise point positioning (PPP) strategy. Using the wall points in and outside the radome, the GNSS-PPP solution was transferred by manual terrestrial measurements to the points inside the radome. Thus, the local network was nearly aligned to a global geocentric reference frame. The wall points were excluded as datum points, because these points were not always visible at each epoch, but these points were used for improving the network geometry.

The introduced a priori stochastic model of the coordinatebased bundle adjustment depends on experience, preliminary investigation, and the accuracy class of the instrument. In addition to the geometric part $\mathbf{Q}_{\mathbf{p p}}$ of the stochastic model, a time-dependent part $\mathbf{Q}_{\mathrm{s}}$ was introduced. The empirical covariance function, cf. Eq. 35, was derived by the recorded $48 \mathrm{~h}$ measurement series (cf. Sect. 9). Due to small data gaps, average values of about $5 \mathrm{~min}$ were used to estimate the empirical auto-covariance function of the time series. The suitable function

$f(\Delta)=u_{a} e^{-u_{b}|\Delta|} \cos u_{c} \Delta$

was selected by plotting the covariance points of the empirical auto-covariance function. The unknown parameters $u_{a}$, $u_{b}$ and $u_{c}$ of the theoretical covariance function were derived by a least-squares adjustment excluding the value for $\Delta=0$, to take only the course of the signal into account [cf. Mikhail and Ackerman (1976, chp. 14.5.2, pp. 404 ff.)]. Figure 7 depicts the empirical normalized auto-covariance function of the polar observations and the corresponding least-squares fits of a theoretical normalized covariance function.

The polar observations become uncorrelated after latest $1 \mathrm{~h}$. Equation 42 was used to create the variance-covariance matrix of the signal $\mathbf{Q}_{\mathrm{S}}$ for the polar observations of each stand point. The a priori stochastic model of the coordinatebased bundle adjustment of each stand point resulted from 

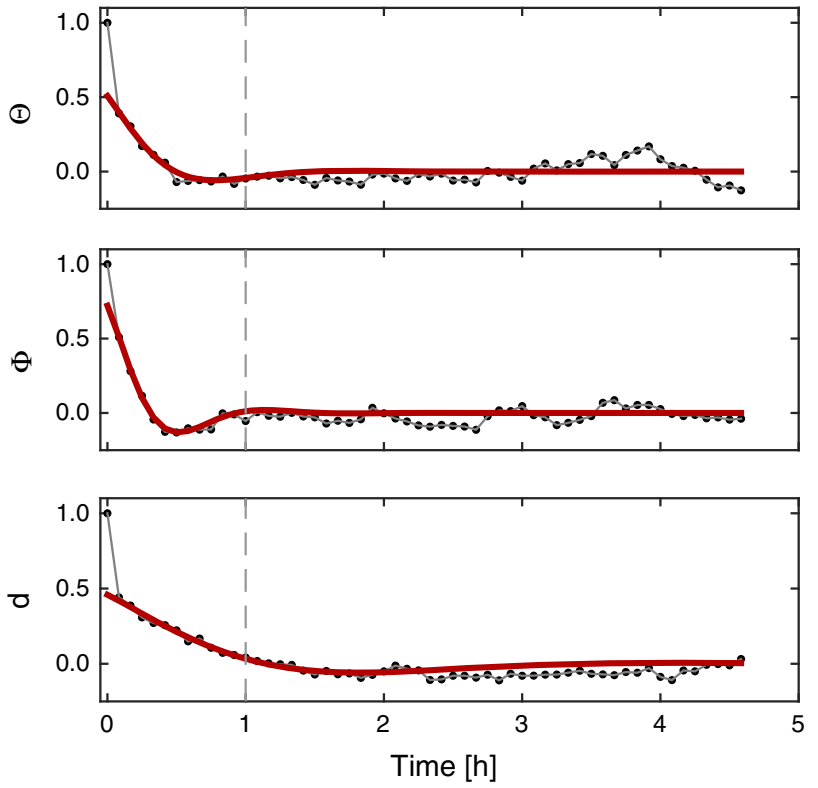

Fig. 7 Empirical normalised auto-covariance functions of the polar observations (black dots) and the corresponding least-squares fits of theoretical normalized covariance functions (red lines)

applying Eq. 34 and was introduced into Eq. 15. Measured points, which were observed by the $j$ th stand point, are correlated to each other by $\mathbf{Q}_{\mathbf{p p}}$ and $\mathbf{Q}_{\mathbf{s}}$. For comparison, in general, the uncertainties of polar observations are considered as being uncorrelated, and the resulting variancecovariance matrix is only a block-diagonal matrix with $3 \times 3$ sub-matrices on the main-diagonal.

The latency time $\delta t_{\text {lat }}$ between the distance measurement and the angular measurements of the total station MS50 is unknown and up to now a correction is impossible. Investigations by Stempfhuber (2004) show a magnitude of up to $\delta t_{\text {lat }}=285 \mathrm{~ms}$ for older Leica instruments. Furthermore, in most cases $\delta t_{\text {lat }}$ is positive, which means that the distance measurement is carried out before the angular measurement [cf. Stempfhuber (2004)]. To take the resulting uncertainties into account, the uncertainty of the latency time $u_{\delta t_{\text {lat }}}=50 \mathrm{~ms}$ was assumed to be uniformly distributed with an expected value $\delta t_{\text {lat }}=300 \mathrm{~ms}$. The influence of $\delta t_{\text {lat }}$ was derived by the Monte Carlo simulation as described in Sect. 5 .

Figure 8 depicts the 3D point coordinate uncertainty caused by the latency time of the total station measurements, derived by Monte Carlo simulations for the first monitoring experiment. The average uncertainty is about $0.025 \mathrm{~mm}$ and values larger than $0.1 \mathrm{~mm}$ are the exception. It can be expected that the latency time of modern instruments is much smaller because of a higher measurement frequency of the angle encoders. Thus, the influence of the latency time becomes smaller and the proposed values are too pessimistic. The variance-covariance matrix of the observed positions at the radio telescope is given by

$\mathbf{Q}_{\mathrm{xyz}}=\mathbf{Q} \mathbf{P P}+\mathbf{Q}_{\text {lat }}$.

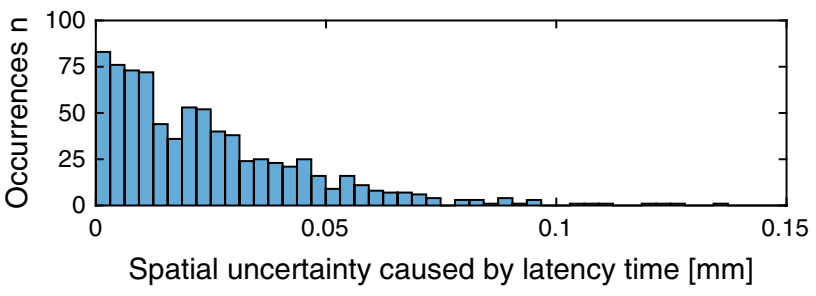

Fig. 8 Histogram of the resulting 3D point coordinate uncertainties caused by latency time of the total station measurements, derived by Monte Carlo simulation for the monitoring experiment of DOY126 (2014)

The average spatial point uncertainty is $2.3 \mathrm{~mm}$. Compared to the spatial uncertainties of the redundantly observed control points, which are below the $1 \mathrm{~mm}$ level, the uncertainty of radio telescope points appears too large. The reasons for the comparatively large value are the observation configuration and the lack of control, the assumed a priori uncertainties in the stochastic model, and the discarding of the variance factor in the coordinate-based bundle adjustment.

As pointed out in Sect. 7, the synchronization error between the total station and the radio telescope system can be expressed as an additional azimuth and elevation angle encoder error. Due to the inaccuracy of computer clocks [cf. Stempfhuber (2004)] the synchronization error is assumed to be $1.5 \mathrm{~s}$, which is a worst-case scenario. Within the recorded timestamps of the total station, time frames were defined and corresponding angle encoder values were selected. These angle encoder values can be interpreted as boundaries of the interval in Eqs. 37 and 38 to derive the uncertainty of the synchronization. Figure 9 depicts the resulting uncertainty of angle encoder readings due to a synchronization error for the first monitoring experiment.
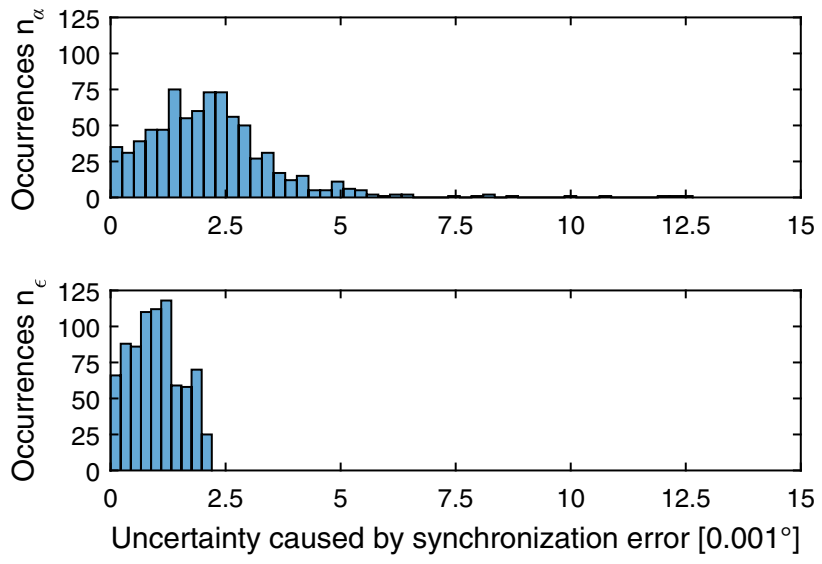

Fig. 9 Histogram of the resulting azimuth $\alpha$ angle (top) and elevation $\epsilon$ angle (bottom) encoder reading uncertainties caused by a clock synchronization error, calculated for the monitoring experiment of DOY 126 (2014) 
Table 3 Overview of the monitoring campaign during CONT14 in May 2014

\begin{tabular}{|c|c|c|c|c|c|c|c|c|c|c|c|}
\hline \multirow{2}{*}{$\begin{array}{l}\text { DOY } \\
(2014)\end{array}$} & \multirow{2}{*}{$\begin{array}{l}\# \\
\text { SP }\end{array}$} & \multicolumn{5}{|c|}{ Instrument stand point on pillar } & \multirow{2}{*}{$\begin{array}{l}\# \\
\text { OP }\end{array}$} & \multirow{2}{*}{$\begin{array}{l}\# \\
\text { SR }(\%)\end{array}$} & \multirow{2}{*}{$\begin{array}{l}\# \\
\text { UP }\end{array}$} & \multicolumn{2}{|c|}{$\underline{\text { DOF }}$} \\
\hline & & 801 & 802 & 803 & 804 & 805 & & & & BA & $\mathrm{RP}$ \\
\hline 126 & 3 & $\bullet$ & $\circ$ & $\bullet$ & $\circ$ & $\bullet$ & 792 & 94.7 & 747 & 78 & 2197 \\
\hline 127 & 3 & $\circ$ & $\bullet$ & $\circ$ & $\bullet$ & $\bullet$ & 825 & 96.6 & 790 & 84 & 2326 \\
\hline 128 & 3 & $\bullet$ & $\circ$ & $\bullet$ & $\bullet$ & $\circ$ & 714 & 85.4 & 667 & 87 & 1957 \\
\hline 129 & 3 & $\circ$ & $\bullet$ & $\bullet$ & $\circ$ & $\bullet$ & 747 & 96.1 & 710 & 69 & 2086 \\
\hline 130 & 3 & $\bullet$ & $\bullet$ & ○ & $\bullet$ & $\circ$ & 826 & 95.9 & 805 & 87 & 2371 \\
\hline 131 & 5 & $\bullet$ & $\bullet$ & $\bullet$ & $\bullet$ & • & 782 & 97.1 & 752 & 63 & 2212 \\
\hline 132 & 3 & $\circ$ & • & - & $\circ$ & • & 778 & 95.1 & 728 & 78 & 2140 \\
\hline 133 & 3 & $\circ$ & $\bullet$ & $\circ$ & • & $\bullet$ & 840 & 95.6 & 804 & 90 & 2368 \\
\hline 134 & 2 & $\circ$ & $\bullet$ & $\circ$ & $\bullet$ & $\circ$ & 599 & 96.2 & 571 & 93 & 1669 \\
\hline 135 & 2 & $\bullet$ & $\circ$ & • & $\circ$ & $\circ$ & 580 & 97.7 & 551 & 63 & 1609 \\
\hline 136 & 3 & $\bullet$ & $\bullet$ & $\circ$ & • & $\circ$ & 831 & 95.9 & 782 & 90 & 2302 \\
\hline 137 & 3 & • & $\circ$ & • & • & $\circ$ & 736 & 95.2 & 706 & 87 & 2074 \\
\hline 138 & 3 & $\bullet$ & $\circ$ & $\bullet$ & $\circ$ & $\bullet$ & 734 & 95.9 & 706 & 81 & 2074 \\
\hline 139 & 2 & $\bullet$ & $\circ$ & • & $\circ$ & $\circ$ & 588 & 93.6 & 569 & 69 & 1663 \\
\hline 140 & 2 & $\circ$ & $\bullet$ & $\circ$ & • & $\circ$ & 658 & 95.2 & 619 & 93 & 1813 \\
\hline \# tooc & & 9 & 9 & 9 & 9 & 7 & & & & & \\
\hline
\end{tabular}

For each of the 15 days information is given on the day of year (DOY), the number of stand points (SP), on which pillar the instrument was placed (filled bullets indicate used pillars), the number of observed points (OP) at the radio telescope, the success rate (SR) of the OP w.r.t. the predicted points of the schedule, the number of used points (UP) at the radio telescope for the reference point determination, the degree of freedom (DOF) for both the bundle adjustment (BA) and the least-squares adjustment of the IVS reference point (RP), and in the last row the number of total occupations (\# tooc) on each survey pillar
Whereas the fraction of the elevation angle is small and always below $0.0025^{\circ}$, the fraction of the azimuth angle holds the larger share on the uncertainty budgeting. The average uncertainty is about $0.002^{\circ}$ and values larger than $0.005^{\circ}$ are the exception. A better classification of this uncertainty results by a conversion into its metric representation. The average distance between the azimuth axis of the radio telescope and the mounted reflectors is about $3 \mathrm{~m}$ and caused a lateral uncertainty of about $0.002^{\circ} \frac{3000 \mathrm{~mm} \pi}{180^{\circ}}=0.1 \mathrm{~mm}$.

The variance-covariance matrices $\mathbf{Q}_{\alpha}$ and $\mathbf{Q}_{\epsilon}$ of the observed radio telescope angles, cf. Sect. 7, were extended by the diagonal matrices $\mathbf{Q}_{\alpha \text {,syn }}$ and $\mathbf{Q}_{\epsilon \text {,syn }}$, respectively. Correlations between the azimuth and elevation angles as well as the coordinates are not assumed, which leads to

$\mathbf{Q}_{\mathrm{xyz}, \alpha, \epsilon}=\left[\begin{array}{ccc}\mathbf{Q}_{\mathrm{xyz}} & \mathbf{0} & \mathbf{0} \\ \mathbf{0} & \mathbf{Q}_{\alpha} & \mathbf{0} \\ \mathbf{0} & \mathbf{0} & \mathbf{Q}_{\epsilon}\end{array}\right]$

The sub-matrices $\mathbf{Q}_{\mathrm{xyz}}, \mathbf{Q}_{\alpha}$ and $\mathbf{Q}_{\epsilon}$ in Eq. 44 are fully populated variance-covariance matrices.

\section{Results of the reference point monitoring}

The data prepared in Sect. 10 were introduced to the IVS reference point determination module of HEIMDALL, cf. Sect. 2. Each epoch was analyzed individually and yielded a daily solution. Height variation caused by changes in temperature were monitored by an invar-wire monitoring system inside the telescope monument, transformed by Eq. 6, and corrected for during the reference point determination. Furthermore, thermal expansions of the elevation cabin were compensated using the recorded monument temperature of the radio telescope and Eqs. 4 and 5.

In total, 15 IVS reference point solutions were derived, cf. Table 3. The degree of freedom of the adjustment process is large, because each point is providing redundant information for Eq. 1 and can be checked for model-compatibility during the reference point determination [cf. Lösler (2009)]. The number of excluded points are given in Table 3.

Table 4 summarizes the 15 individual solutions for the reference point and the estimated axis-offset in a global geodetic reference frame. The variations of the individual solutions are below $1 \mathrm{~mm}$. To combine the individual solutions, a recursive parameter estimation was applied as described in Sect. 8.

A precondition for a stability check of the reference point is that the control points of the local network are stable. Besides the reference point and the axis-offset, the estimates of the control points of the corresponding coordinate-based bundle adjustment were also stacked. These points become important if the geodetic datum has to be changed. The uncertainties were derived by Eq. 9. Therefore, time series for all points were generated and analyzed for deformations. 
Table 4 Overview of the results during CONT14

\begin{tabular}{llllr}
\hline DOY & $x_{\mathrm{RP}}(\mathrm{m})$ & $y_{\mathrm{RP}}(\mathrm{m})$ & $z_{\mathrm{RP}}(\mathrm{m})$ & $e_{\alpha, \epsilon}(\mathrm{m})$ \\
\hline 126 & 3370605.7907 & 711917.7234 & 5349830.9111 & -0.0056 \\
127 & 3370605.7904 & 711917.7236 & 5349830.9111 & -0.0058 \\
128 & 3370605.7910 & 711917.7234 & 5349830.9111 & -0.0058 \\
129 & 3370605.7897 & 711917.7238 & 5349830.9110 & -0.0058 \\
130 & 3370605.7902 & 711917.7238 & 5349830.9107 & -0.0060 \\
131 & 3370605.7904 & 711917.7238 & 5349830.9112 & -0.0059 \\
132 & 3370605.7903 & 711917.7236 & 5349830.9113 & -0.0058 \\
133 & 3370605.7904 & 711917.7234 & 5349830.9107 & -0.0059 \\
134 & 3370605.7901 & 711917.7237 & 5349830.9107 & -0.0060 \\
135 & 3370605.7902 & 711917.7237 & 5349830.9115 & -0.0058 \\
136 & 3370605.7901 & 711917.7242 & 5349830.9107 & -0.0059 \\
137 & 3370605.7904 & 711917.7231 & 5349830.9111 & -0.0059 \\
138 & 3370605.7904 & 711917.7235 & 5349830.9108 & -0.0059 \\
139 & 3370605.7903 & 711917.7238 & 5349830.9108 & -0.0058 \\
140 & 3370605.7902 & 711917.7241 & 5349830.9106 & -0.0060 \\
Filter solution & 3370605.7903 & 711917.7236 & 5349830.9110 & -0.0059 \\
$95 \%$ confidence level & 0.0003 & 0.0002 & 0.0004 & 0.0001 \\
\hline
\end{tabular}

For each of the 15 days information is given on the day of year (DOY), the geocentric coordinates of the IVS reference point $\left(x_{\mathrm{RP}}, y_{\mathrm{RP}}, z_{\mathrm{RP}}\right)$, and the axis-offset $e_{\alpha, \epsilon}$ of the Onsala $20 \mathrm{~m}$ telescope. The uncertainties of the individual results are on the order of $0.3,0.2$ and $0.4 \mathrm{~mm}$ for the $x$-, $y$ - and $z$-component, respectively, and $0.1 \mathrm{~mm}$ for the axis-offset. The filter solution based on the combination of the 15 days and its $95 \%$ confidence level is given in the last two rows
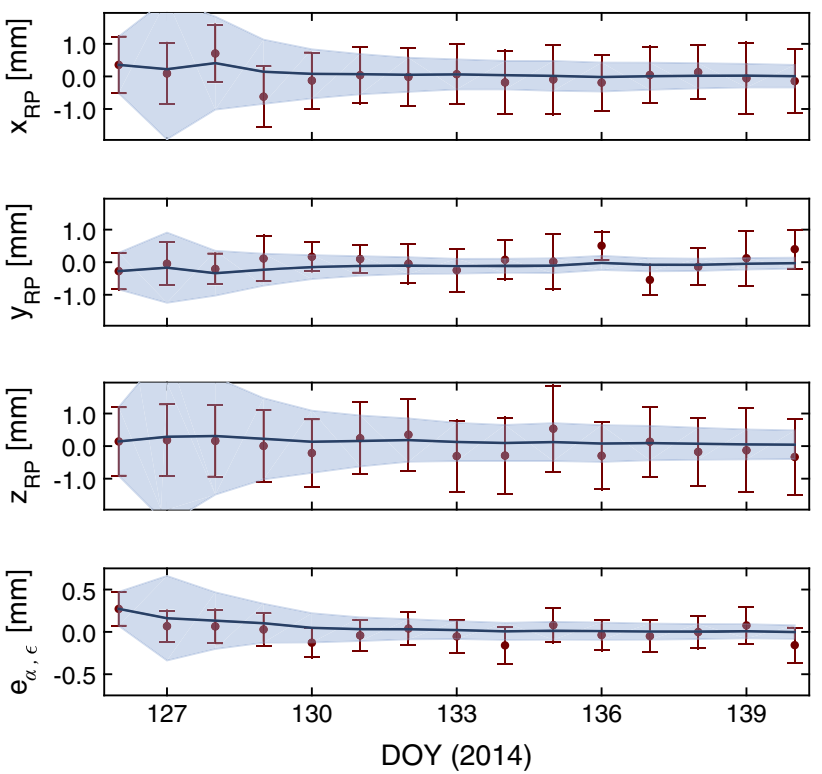

Fig. 10 Daily individual solutions (red, with error bars) for the reference point coordinates and the axis-offset and the corresponding recursive filter results (blue solid line). The blue-grey uncertainty band relates to the $95 \%$ confidence level

Figure 10 depicts the individual solutions together with the filtered solution derived by recursive parameter estimation, and proves the stability of the reference point position. The uncertainties of the geocentric $x_{\mathrm{RP}}$ and $z_{\mathrm{RP}}$ components are slightly larger than for $y_{\mathrm{RP}}$. An analysis of the variancecovariance matrices shows a large dependency between the $x_{\mathrm{RP}}$ and $z_{\mathrm{RP}}$ components. The estimated correlation coefficient is $\rho_{x_{\mathrm{RP}}, z_{\mathrm{RP}}} \approx 0.8$ and results from the selected global geodetic datum. In a topocentric reference frame the uncertainties of the $x_{\mathrm{RP}}$ and $y_{\mathrm{RP}}$ components are on the same order of magnitude, because of the surrounding symmetric observation configuration, cf. Table 3.

A datum-independent specification of the uncertainties can be derived by a spectral decomposition of the variance-covariance matrix [e.g. Mikhail and Ackerman (1976, chp. A6., pp. 451 ff.), Koch (1999, chp. 1.4.2, pp. 44 ff.)], i.e.

$\mathbf{Q}_{\mathrm{RP}}=\mathbf{V D V}^{\mathrm{T}}$.

Here, $\mathbf{V}$ and $\mathbf{D}$ contain the eigenvectors and eigenvalues of the matrix $\mathbf{Q}_{\mathrm{RP}}$, respectively, and represent the orientation and the dimension of the spatial hyperellipsoid. The maximum eigenvalue and the corresponding eigenvector specify the magnitude and the direction of the largest point uncertainty of the reference point. The maximum uncertainty of the filtered reference point is $u_{\max , 95 \%}=0.5 \mathrm{~mm}$ (95\% confidence level) and is a better representation of the achieved uncertainties. Moreover, the memory effect of the filter results in a smooth curve progression. Whereas standard epoch-based determination of a reference point only uses observations 
of one particular survey epoch, the recursive parameter estimation involves all prior information. Small variations of the individual solution are attenuated and the robustness of the solution is increased. The recursive estimates fulfill the requirements of $1 \mathrm{~mm}$ [cf. Rothacher et al. (2009)]. Independent of the single-point uncertainty, reliable results can be expected, if a homogenous point distribution is achieved and any kind of extrapolation is prevented.

\section{Summary and conclusion}

A terrestrial monitoring campaign was carried out at the Onsala Space Observatory during the 15-day-long global VLBI campaign CONT14. A part of the local site network at Onsala as well as reflectors mounted on the $20 \mathrm{~m}$ radio telescope were observed during ongoing normal telescope operations. Based on these observations, in total 15 individual solutions for the radio telescope reference point were derived. In contrast to prior investigations, a newly developed coordinate-based bundle adjustment was applied for the data analysis. The advanced stochastic model of the adjustment process is based on the calibration model proposed by Hughes et al. (2011) and regards more than 25 parameters [cf. Lösler et al. (2015a)]. Moreover, the uncertainty budgeting was further extended by additional timedependent covariances and it also regards the persistence of the observations in case of high-frequency measurements. The functional model of the network adjustment is based on a bundle adjustment that is detached from the local gravity field [cf. Lösler and Eschelbach (2012)]. To avoid an over-parametrization, the number of additional parameters of the functional model of the bundle adjustment was evaluated using the information criteria technique AIC. It could be shown that the 6-DOF solution of the bundle adjustment was preferred even if the extent of the network is small.

Whereas in conventional approaches the global transformation process between local and global coordinate frames is carried out as a final step, in our approach the transformation into a global reference frame takes place right in the beginning of the bundle adjustment. Combination of GNSS observations and classic terrestrial measurements in a global reference frame permits an adjustment process directly in the target system, e.g. the ITRF. The bundle adjustment of the radome network as well as the reference point determination was carried out in a true Cartesian global reference frame. The uncertainties of the angle encoders of the radio telescope were modeled by Fourier series, which provides full variance-covariance matrices for the azimuth and elevation angles. Due to the continuously moving radio telescope during CONT14, additional uncertainties had to be taken into account. The influence of timing errors was studied and considered. The 15 individual solutions of the automated and continual monitoring process were combined by recursive parameter estimation as firstly proposed by Lösler et al. (2013). Due to the relatively short observation period of just 15 days, an additional variance matrix for the process noise was discarded. However, for longer observation periods it would have to be taken into account. The detected variations of the IVS reference point during the CONT14 campaign are on the sub-millimeter level and the smoothed filter results show a straight curve profile.

The GGOS strives for sub-millimeter accuracy and continual determinations of the reference points of space geodetic instruments [cf. Rothacher et al. (2009)]. The concept presented in this work fulfills these requirements even during normal operation of the radio telescope. It thus appears a very promising and valuable approach for the upcoming VGOS network with planned 24/7 operations (Petrachenko et al. 2009) and it should be easily possible to extend the concept also to VGOS twin telescopes [cf. Neidhardt et al. (2011), Haas (2013)].

Future work will focus on an improved integration of our system with the radio telescope control to reduce the uncertainty budgeting caused by timing errors between the radio telescope and the observation instrument. The latency time should also be taken into account and the application of mobile laser trackers will be evaluated. This type of instrument has a high internal measurement frequency which promises to be beneficial in particular for the observation of moving targets as, e.g. reflectors mounted on a radio telescope in operation. Moreover, the supported reflectors of a laser tracker are in general glassless and thus the lateral and radial errors as depicted in Fig. 4 become obsolete. Finally, the collaboration of classical geodetic survey and GNSSbased reference point determinations will be addressed.

Acknowledgments We thank Tobias Wulf and Johann Elter for supporting this research by their Bachelor's theses. We thank Lars Wennerbäck, Christer Hermansson and Ronny Wingdén from the mechanical workshop at the observatory for their support drilling holes in the radome foundation wall and installing the magnetic reflector holders that were used to connect the networks inside and outside the radome building.

Open Access This article is distributed under the terms of the Creative Commons Attribution 4.0 International License (http://creativecomm ons.org/licenses/by/4.0/), which permits unrestricted use, distribution, and reproduction in any medium, provided you give appropriate credit to the original author(s) and the source, provide a link to the Creative Commons license, and indicate if changes were made.

\section{Appendix 1: Brief derivation of a compensation model for polar measurement instruments}

Polar measurement instruments like total stations, laser scanners or laser trackers consist of three axes, namely the yaw 
axis, the pitch axis and the axis of the laser beam. The polar observations $\hat{d}, \hat{\Theta}$ and $\hat{\Phi}$ are transformed into a Cartesian representation w.r.t. the position and orientation of these axes.

The axes of the instrument can be parametrized by the vector equation of a straight spatial line, i.e. by a location point and a direction vector w.r.t. the instrument fixed frame. Defining an initial position, i.e. $\hat{\Theta}=0$ and $\hat{\Phi}=\frac{\pi}{2}$, it is assumed that the yaw axis is aligned to the $z$-axis, i.e. the location point is the origin of the coordinate system and the direction vector is $\mathbf{n}_{\text {yaw }}=\left(\begin{array}{lll}0 & 0 & 1\end{array}\right)^{\mathrm{T}}$, the pitch axis and the beam axis are parallel to the $y z$-plane and to the $x y$-plane, respectively. The location point of the pitch axis is shifted by the axis offset $e_{\Theta} \Phi$, i.e.

$\mathbf{e}_{\Theta, \Phi}=\left(\begin{array}{c}e_{\Theta, \Phi} \\ 0 \\ 0\end{array}\right)$

The direction of the pitch axis is biased by the trunnion axis error $\kappa$, i.e.

$\mathbf{n}_{\mathbf{e} \Theta, \Phi}=\mathbf{R}_{\kappa}^{x}\left(\begin{array}{l}0 \\ 1 \\ 0\end{array}\right)$.

The axis of the laser beam is shifted by the coordinates of the distance measurement unit, i.e.

$\mathbf{b}_{0}=\left(\begin{array}{l}t_{d, x} \\ t_{d, y} \\ t_{d, z}\end{array}\right)$,

and the direction is biased by the horizontal collimation error $v$, i.e.

$\mathbf{n}_{\mathbf{0}}=\mathbf{R}_{v}^{z}\left(\begin{array}{l}1 \\ 0 \\ 0\end{array}\right)$.

In Eq. 23 the slope distance is corrected by a scaling factor $\mu$ and a displacement offset $\lambda$. Obviously, the displacement error of the laser beam $t_{d, x}$ can be omitted because this error can also be compensated by $\lambda$. In this case $\lambda$ compensates both, the displacement offset of the measuring beam and the $x$-component of the displaced distance measurement unit.

If the telescope of the instrument is rotated by an angle $\hat{\Theta}$ around the yaw axis, the location points of the pitch axis and the beam axis become $\mathbf{R}_{\hat{\Theta}}^{z} \mathbf{e}_{\Theta, \Phi}$ and $\mathbf{R}_{\hat{\Theta}}^{z} \mathbf{b}_{\mathbf{0}}$, respectively. A further rotation by the angle $\hat{\Phi}$ around the pitch axis must be parametrized by a rotation sequence, because this rotation can only be applied in the initial position, i.e. the pitch axis must be aligned to the $y$-axis of the instrument frame:

$\mathbf{R}=\mathbf{R}_{\hat{\Theta}}^{z} \mathbf{R}_{\kappa}^{x} \mathbf{R}_{\hat{\Phi}-\frac{\pi}{2}}^{y} \mathbf{R}_{-\kappa}^{x} \mathbf{R}_{-\hat{\Theta}}^{z}$
By applying Eq. 50 to the location point of the laser beam, the rotated position becomes

$\mathbf{b}(\hat{\Theta}, \hat{\Phi})=\mathbf{R}_{\hat{\Theta}}^{z} \mathbf{e}_{\Theta, \Phi}+\mathbf{R} \mathbf{R}_{\hat{\Theta}}^{z}\left(\mathbf{b}_{\mathbf{0}}-\mathbf{e}_{\Theta, \Phi}\right)$,

which is equivalent to Eq. 27. Analogously, the rotated direction of the laser beam is obtained by

$\mathbf{n}(\hat{\Theta}, \hat{\Phi})=\mathbf{R} \mathbf{R}_{\hat{\Theta}}^{z} \mathbf{n}_{\mathbf{0}}$

which is equivalent to Eq. 28. By adding the distance $\hat{d}$, Eqs. 27 and 28 as well as Eqs. 51 and 52 define a straight spatial line that converts the polar observation into its Cartesian representation, w.r.t. the instrument frame, cf. Eq. 26. The interesting reader is referred to the work of Hughes et al. (2011) to get a detailed description of the compensation model.

\section{References}

Adunka F (2007) Messunsicherheiten: Theorie und Praxis, Vulkan, Essen, 3rd edn

Akaike H (1974) A new look at the statistical model identification. IEEE Trans Autom Control 19:716-723

Altamimi Z, Xavier Collilieux, Métivier L (2011) ITRF2008: an improved solution of the international terrestrial reference frame. J Geod 85:457-473. doi:10.1007/s00190-011-0444-4

Awange JL, Grafarend EW (2005) Solving algebraic computational problems in geodesy and geoinformatics. Springer, Heidelberg/Berlin

Bähr HG, Richter R (1975) Über die Wahl von a-priori-Korrelationen. Zeitschrift für Vermessungswesen 100:180-188

Burnham KP, Anderson DR (2002) Model selection and multimodel inference - a practical information-theoretic approach, 2nd edn. Springer, Heidelberg/Berlin

Calkins JM (2002) Quantifying coordinate uncertainty fields in coupled spatial measurement systems. Dissertationen, Faculty of the Virginia Polytechnic Institute and State University, Virginia

Ciddor P (2002) Refractive index of air: 3. The role of CO2, H2O, and refractivity virials. Appl Opt 41:2292-2298. doi:10.1364/AO.41. 002292

Claeskens G, Hjort NL (2008) Model selection and model averaging. Cambridge series in statistical and probabilistic mathematics. Cambridge University Press, Cambridge

Dawson J, Sarti P, Johnston G, Vittuari L (2007) Indirect approach to invariant point determination for SLR and VLBI systems: an assessment. J Geod 81:433-441. doi:10.1007/s00190-006-0125-x

DIN 1319 (1995) Grundlagen der Meßtechnik. Berlin, Beuth

Eschelbach C, Haas R (2003) The IVS-reference point at onsala-high end solution for a real 3D-determination. In: Schwegmann W, Thorandt V Proceedings of the 16th Working Meeting on European VLBI for Geodesy and Astronomy, pp 109-118

Grimm DE (2014) Flexibility in choice of methods with Leica Nova MS50 (in German). Allgemeine Vermessungs-Nachrichten 121:326-331

Grimm DE, Hornung U (2015) Leica ATRplus improved performance for automated target aiming and locking (in German). Allgemeine Vermessungs-Nachrichten 122:269-276

GUM (2008a) Evaluation of measurement data-guide to the expression of uncertainty in measurement. JCGM 100:2008, GUM 1995 
with minor corrections. http://www.bipm.org/en/publications/ guides/gum.html. Retrieved 4 June 2015

GUM (2008b) Evaluation of measurement data-supplement 1 to the "Guide to the expression of uncertainty in measurement"propagation of distributions using a Monte Carlo method. JCGM 101:2008. http://www.bipm.org/en/publications/guides/ gum.html. Retrieved 4 June 2015

Haas R (2013) The Onsala twin telescope project. In: Zubko N, Poutanen M Proceedings of the 21st Meeting of the European VLBI Group for Geodesy and Astronomy, Reports of the Finnish Geodetic Institute, vol 1, pp 61-66

Haas R, Nothnagel A, Schuh H, Titov O (1999) Explanatory supplement to the section 'Antenna Deformation' of the IERS Conventions (1996). In: Schuh H DGFI report nr. 71, Deutsches Geodätisches Forschungsinstitut (DGFI), Munich, pp 26-29

Heck B (2003) Rechenverfahren und Auswertemodelle der Landesvermessung: Klassische und moderne Methoden, 3rd edn. Wichmann, Heidelberg

Hennes M (2007) Konkurrierende Genauigkeitsmaße Potential und Schwächen aus der Sicht des Anwenders. Allgemeine Vermessungs-Nachrichten 7:136-146

Heunecke H, Kuhlmann H, Welsch W, Eichhorn A, Neuner H (2013) Handbuch Ingenieurgeodäsie: Auswertung geodätischer Überwachungsmessungen, 2nd edn. Wichmann, Heidelberg

Höpcke W (1980) Fehlerlehre und Ausgleichungsrechnung. de Gruyter, Berlin/New York

Hughes B, Forbes A, Lewis A, Sun W, Veal D, Nasr K (2011) Laser tracker error determination using a network measurement. Meas Sci Technol 22:1-12

Kallio U, Poutanen M (2012) Can we really promise a mm-accuracy for the local ties on a Geo-VLBI antenna. In: Geodesy for Planet Earth, International Association of Geodesy Symposia, vol 136. Springer, Heidelberg/Berlin, pp 35-42. doi:10.1007/978-3-642-20338-1_5

Kalman RE (1960) A new approach to linear filtering and prediction problems. Transaction of the ASME. J Basic Eng 82:35-45

Koch KR (1999) Parameter Estimation and hypothesis testing in linear models, 2nd edn. Springer, Heidelberg/Berlin

Koch KR (2007) Introduction to bayesian statistics, 2nd edn. Springer, Heidelberg/Berlin

Koch KR (2014) Robust estimations for the nonlinear Gauss Helmert model by the expectation maximization algorithm. J Geod 88:263271. doi:10.1007/s00190-013-0681-9

Krickel B (2004) Leistungskriterien zur Qualitätskontrolle von Robottachymetern. Dissertationen, Mitteilungen aus den Geodätischen Instituten der Rheinischen Friedrich-Wilhelms-Universität, vol 92. Bonn

Kuhlmann H (2003) Kalman-filtering with coloured measurement noise for deformation analysis. In: Proceedings of the 11th FIG symposium on deformation measurements, Santorini

Lanotte R, Pirri M, Bianco G (2008) Matera site survey and VLBI invariant point determination. In: Finkelstein A, Behrend D (eds) Proceedings of the 5th IVS general meeting-measuring the future. St. Petersburg, pp 87-92

Lehmann R (2014) Transformation model selection by multiple hypothesis testing. J Geod 88:1117-1130. doi:10.1007/ s00190-014-0747-3

Lehmann R, Lösler, M (2016) Multiple outlier detection-hypothesis tests versus model selection by information criteria. J Surv Eng (in-print)

Leinen S, Becker M, Dow J, Feltens J, Sauermann K (2007) Geodetic determination of radio telescope antenna reference point and rotation axis parameters. J Surv Eng 133:41-51. doi:10.1061/ (ASCE)0733-9453(2007)133:2(41)

Lewis A, Hughes B, Forbes A, Sun W, Veal D, Nasr K (2011) Determination of misalignment and angular scale errors of a laser tracker using a new geometric model and a multi-target network approach.
In: Proceedings of the macroScale 2011-recent developments in traceable dimensional measurements, Federal Office of Metrology METAS, Wabern. doi:10.7795/810.20130620S

Li J, Xiong F, Yu C, Zhang J, Guo L, Fan Q (2014) Precise determination of the reference point coordinates of Shanghai Tianma 65-m radio telescope. Chin Sci Bull 59:2558-2567. doi:10.1007/ s11434-014-0349-8

Lienhart W, Zogg HM, Nindl D (2009) Innovative Lösungen zur Erreichung höchster Genauigkeit und Geschwindigkeit am Beispiel der TS30 Totalstation von Leica Geosystems. Allgemeine Vermessungs-Nachrichten 116:374-381

Lösler M (2008) Reference point determination with a new mathematical model at the $20 \mathrm{~m}$ VLBI radio telescope in Wettzell. J Appl Geod 2:233-238. doi:10.1515/JAG.2008.026

Lösler M (2009) New mathematical model for reference point determination of an azimuth-elevation type radio telescope. J Surv Eng 135:131-135. doi:10.1061/(ASCE)SU.1943-5428.0000010

Lösler M, Haas R, Eschelbach C (2013) Automated and continual determination of radio telescope reference points with sub-mm accuracy: results from a campaign at the Onsala Space Observatory. J Geod 87:791-804. doi:10.1007/s00190-013-0647-y

Lösler M, Eschelbach C (2012) Concept of a realisation of a prototype for adequate evaluation of polar measurements (in German). Allgemeine Vermessungs-Nachrichten 119:249-258

Lösler M, Eschelbach C (2014) To determine the parameters of a spatial affine transformation (in German). Allgemeine VermessungsNachrichten 121:273-277

Lösler M, Eschelbach C, Haas R (2015a) Coordinate based bundle adjustment-advanced network adjustment model for polar measurement systems. In: Haas R, Colomer F (eds) Proceedings of the 22th Working Meeting on European VLBI for Geodesy and Astronomy. Ponta Delgada, Azores, pp 135-139

Lösler M, Arnold M, Bähr H, Eschelbach C, Bahlo T, Grewe R, Hug F, Jürgensen L, Winkemann P, Pietralla N (2015b) Highprecision surveying of the beam-line elements of the electron linear accelerator S-DALINAC (in German). Zeitschrift für Geodäsie, Geoinformatik und Landmanagement 140:346-356. doi:10. 12902/zfv-0090-2015

Lossin T, Lösler M, Neidhardt A, Lehmann R, Mähler S (2014) The usage of recursive parameter estimation in automated reference point determination. In: Behrend $\mathrm{D}$, Baver KD, Armstrong KL (eds) Proceedings of the 8th IVS General Meeting-VGOS: the New VLBI Network, Shanghai, pp 344-348

Mikhail EM, Ackerman F (1976) Observations and least squares. University Press of America, New York

Muralikrishnan B, Sawyer DS, Blackburn CJ, Phillips SD, Borchardt BR, Estler WT (2009) ASME B89.4.19 performance evaluation tests and geometric misalignments in laser trackers. J Res Natl Inst Stand Technol 114:21-35

Neidhardt A, Kronschnabl G, Klügel T, Hase H, Pausch K, Göldi W (2011) VLBI2010 — current status of the TWIN radio telescope project at Wettzell, Germany. In: Alef W, Bernhart S, Nothnagel A (eds) Proceedings of the 21th Working Meeting on European VLBI for Geodesy and Astronomy, Bonn, pp 67-70

Neitzel F (2010) Generalization of total least-squares on example of unweighted and weighted 2D similarity transformation. J Geod 84:751-762. doi:10.1007/s00190-010-0408-0

Ning T, Haas R, Elgered G (2015) Determination of the local tie vector between the VLBI and GNSS reference points at Onsala using GPS measurements. J Geod 89:711-723. doi:10.1007/ s00190-015-0809-1

Nothnagel A (2009) Conventions on thermal expansion modelling of radio telescopes for geodetic and astrometric VLBI. J Geod 83:787-792. doi:10.1007/s00190-008-0284-z

Pauli W (1969) Vorteile eines kippbaren Reflektors bei der elektrooptischen Streckenmessung. Vermessungstechnik 17:412-415 
Petrachenko B, Niell A, Behrend D, Corey B, Böhm J, Charlot P, Collioud A, Gipson J, Haas R, Hobiger T, Koyama Y, MacMillan D, Malkin Z, Nilsson T, Pany A, Tuccari G, Whitney A, Wresnik J (2009) Design aspects of the VLBI2010 system, NASA, NASA/TM-2009-214180, Washington, DC

Rothacher M, Beutler G, Behrend D, Donnellan A, Hinderer J, Ma C, Noll C, Oberst J, Pearlman M, Plag H-P, Richter B, Schöne T, Tavernier G, Woodworth PL (2009) The future global geodetic observing system. In: Plag H-P, Pearlman M The global geodetic observering system. meeting the requirements of a global society on an changing planet in 2020. Springer, Heidelberg/Berlin, pp 237-272. doi:10.1007/978-3-642-02687-4

Rüeger JM (1996) Electronic distance measurement-an introduction, 4rd edn. Springer, Heidelberg/Berlin

Sarti P, Sillard P, Vittuari L (2004) Surveying co-located space-geodetic instruments for ITRF computation. J Geod 78:210-222. doi:10. 1007/s00190-004-0387-0

Schwarz W (1994) Zur Reduktion der Messungen bei räumlichen Punktbestimmungen. Allgemeine Vermessungs-Nachrichten 101:207218

Shen YZ, Chen Y, Zheng DH (2006) A quaternion-based geodetic datum transformation algorithm. J Geod 80:233-239. doi:10.1007/ s00190-006-0054-8

Sovers OJ, Fanselow JL, Jacobs CS (1998) Astrometry and geodesy with radio interferometry: experiments, models, results. Rev Mod Phys 70(4):1393-1454. doi:10.1103/RevModPhys.70.1393
Stempfhuber WV (2004) Ein integritätswahrendes Messsystem für kinematische Anwendungen. Dissertationen, Bayerische Akademie der Wissenschaften, Deutsche Geodätische Kommission, Reihe C, vol 309, München

Taubenheim J (1969) Geophysikalische Monographien: Statistische Auswertung geophysikalischer und meteorologischer Daten. Akademische Verlagsgesellschaft Geest and Portig K.-G, Leipzig

Teunissen PJG (2009) Dynamic data processing-recursive leastsquares. VSSD, Delft

United Nations (2015) Resolution on A global geodetic reference frame for sustainable development. Sixy-ninth session, Agenda item 9, A/69/L.53. http://www.un.org/ga/search/view_doc.asp? symbol=A/69/L.53. Retrieved 4 June 2015

Wresnik J, Haas R, Böhm J, Schuh H (2007) Modeling thermal deformation of VLBI antennas with a new temperature model. J Geod 81:423-431. doi:10.1007/s00190-006-0120-2

$\mathrm{Xu} \mathrm{P}$ (2013) The effect of incorrect weights on estimating the variance of unit weight. Stud Geophys Geod 57:339-352. doi:10.1007/ s11200-012-0665-x 